TRANSACTIONS OF THE

AMERICAN MATHEMATICAL SOCIETY

Volume 357, Number 3, Pages 1061-1093

S 0002-9947(04)03536-6

Article electronically published on July 16, 2004

\title{
PARAMETRIC BÄCKLUND TRANSFORMATIONS I: PHENOMENOLOGY
}

\author{
JEANNE N. CLELLAND AND THOMAS A. IVEY
}

\begin{abstract}
We begin an exploration of parametric Bäcklund transformations for hyperbolic Monge-Ampère systems. (The appearance of an arbitrary parameter in the transformation is a feature of several well-known completely integrable PDEs.) We compute invariants for such transformations and explore the behavior of four examples, two of which are new, in terms of their invariants, symmetries, and conservation laws. We prove some preliminary results and indicate directions for further research.
\end{abstract}

\section{INTRODUCTION}

In this paper we will study parametric Bäcklund transformations between secondorder hyperbolic Monge-Ampère equations for one function of two variables. These are partial differential equations (PDEs) of the form

$$
A\left(z_{x x} z_{y y}-z_{x y}^{2}\right)+B z_{x x}+2 C z_{x y}+D z_{y y}+E=0
$$

where the coefficients $A, B, C, D, E$ are functions of the variables $x, y, z, z_{x}, z_{y}$. The equation is hyperbolic if it has distinct, real characteristics at each point, i.e., if $A E-B D+C^{2}>0$.

Before giving a formal definition, we consider the following classical example.

Example. Consider the pair of PDEs

$$
\begin{aligned}
& z_{x}-\bar{z}_{x}=\lambda \sin (z+\bar{z}), \\
& z_{y}+\bar{z}_{y}=\frac{1}{\lambda} \sin (z-\bar{z})
\end{aligned}
$$

for functions $z(x, y), \bar{z}(x, y)$, with $\lambda$ a nonzero real number. By differentiating the first equation with respect to $y$ and the second equation with respect to $x$ and then adding or subtracting the two equations, it can be shown that any smooth functions $z(x, y), \bar{z}(x, y)$ satisfying these equations must both be solutions of the sine-Gordon equation

$$
z_{x y}=\frac{1}{2} \sin (2 z)
$$

From another point of view, if $z(x, y)$ is a known solution of (2), then (1) is a compatible, overdetermined system for $\bar{z}(x, y)$. Solving this system, which only requires integrating ordinary differential equations, yields new solutions of (1) which

Received by the editors May 8, 2003 and, in revised form, September 4, 2003.

2000 Mathematics Subject Classification. Primary 37K35, 58J72; Secondary 35L10, 53C10, $58 \mathrm{~A} 15$.

Key words and phrases. Bäcklund transformations, hyperbolic Monge-Ampère systems, Weingarten surfaces, exterior differential systems, Cartan's method of equivalence.

(C)2004 American Mathematical Society 
are Bäcklund transformations of the original solution. For example, taking $z(x, y)=$ 0 yields

$$
\bar{z}(x, y)=\tan ^{-1}\left(e^{-\left(\lambda x+\frac{1}{\lambda} y+c\right)}\right) .
$$

These are the 1-soliton solutions of the sine-Gordon equation.

The sine-Gordon equation is a classical example of an integrable system. The remarkable features typical of such systems include an infinite sequence of conservation laws, a method of solution by inverse scattering [7], and Bäcklund transformations. (Indeed, the most interesting examples of Bäcklund transformations come from integrable systems.) Note that the arbitrary parameter $\lambda$ appearing in the Bäcklund transformation (11) does not appear in the sine-Gordon equation itself. This parameter plays a critical role in the theory of Bäcklund transformations of integrable systems; for instance, it is possible to derive the infinite hierarchy of conservation laws for the sine-Gordon equation by performing a series expansion of the equations (11) in terms of $\lambda$ [18. Furthermore, (1) is equivalent to the sineGordon AKNS system (with $\lambda$ as spectral parameter), which is the centerpiece of the solution by inverse scattering [15].

Remark. The system (10) is an auto-Bäcklund transformation for sine-Gordon. In general, Bäcklund transformations can take solutions of one PDE and produce solutions to a different PDE. For example, suppose that $z(x, t)$ is a solution of the nonlinear heat equation $z_{t}=\left(f(z) z_{x}\right)_{x}$. If $y(x, t)$ is a solution to the equations

$$
y_{x}=z, \quad y_{t}=f(z) z_{x}
$$

and we take $\bar{z}=1 / z$ to be a function of $y$ and $t$, then $\bar{z}$ is a solution to another nonlinear heat equation $\bar{z}_{t}=\left(\bar{f}(\bar{z}) \bar{z}_{y}\right)_{y}$, where $\bar{f}$ is related to $f$ by $z f(z)=\bar{z} \bar{f}(\bar{z})$. This example also shows that, in general, Bäcklund transformations may involve a change of independent variable.

As in [6, we will generalize the notion of a Monge-Ampère equation slightly, considering Bäcklund transformations between certain exterior differential systems known as Monge-Ampère systems.

An exterior differential system (EDS) on a manifold $\mathcal{M}$ is a differentially closed ideal $\mathcal{I}$ in the graded algebra, under wedge product, of differential forms on $\mathcal{M}$. Any system of partial differential equations can be formulated as an EDS $\mathcal{I}$, and solutions of the PDE system correspond to integral manifolds of $\mathcal{I}$, i.e., submanifolds $\mathcal{N} \subset \mathcal{M}$ which satisfy the condition that all the forms in $\mathcal{I}$ vanish when pulled back to $\mathcal{N}$.

Definition 1.1. A Monge-Ampère system $\mathcal{I}$ is an EDS on a 5 -dimensional manifold $\mathcal{M}$ such that $\mathcal{I}$ is locally generated by a contact form $\theta$ (i.e., a 1 -form $\theta$ with the property that $\theta \wedge d \theta \wedge d \theta \neq 0$ ), the 2 -form $\Theta=d \theta$, and another 2 -form $\Psi$ which is linearly independent from $\Theta$ and wedge products involving $\theta$.

A Monge-Ampère system $\mathcal{I}$ is hyperbolic if the homogeneous quadratic equation

$$
(\mu \Theta+\nu \Psi) \wedge(\mu \Theta+\nu \Psi) \equiv 0 \bmod \theta
$$

has distinct real roots with homogeneous coordinates $\left[\mu_{i}, \nu_{i}\right], i=1,2$. This condition agrees with the traditional definition of hyperbolicity, and it implies that there are two independent linear combinations $\mu_{i} \Theta+\nu_{i} \Psi$ which are decomposable modulo $\theta$; i.e., they are each congruent modulo $\theta$ to a wedge product of a pair of 
1-forms. Associated to $\mathcal{I}$ are two Monge characteristic systems, each containing the factors of one of these decomposable 2-forms, together with $\theta$. (The characteristic systems must each contain $\theta$ because the factors of the decomposable 2 -forms are only well defined modulo $\theta$.)

Remark. Those EDS which are generated algebraically by 1-forms and their exterior derivatives are known as Pfaffian systems; the dimension of the space of 1-forms in the system is the rank of the system. Any hyperbolic Monge-Ampère system is equivalent, under prolongation, to a type of rank three Pfaffian system known as a hyperbolic exterior differential system [3].

Example (cont'd). The sine-Gordon equation (2) may be described as a hyperbolic Monge-Ampère system $\mathcal{I}$ on $\mathbb{R}^{5}$ (with coordinates $(x, y, z, p, q)$ ) generated by the forms

$$
\begin{gathered}
\theta=d z-p d x-q d y, \\
\Theta=-d p \wedge d x-d q \wedge d y, \\
\Psi=\left[d p-\frac{1}{2} \sin (2 z) d y\right] \wedge d x .
\end{gathered}
$$

Two-dimensional integral manifolds of this system that satisfy the independence condition $d x \wedge d y \neq 0$ are naturally in one-to-one correspondence with solutions of (2).

Note that $\Psi$ is decomposable; another decomposable linear combination of $\Psi$ and $\Theta$ is $\Psi+\Theta=-\left[d q-\frac{1}{2} \sin (2 z) d x\right] \wedge d y$. Thus, the two characteristic systems are

$$
\begin{aligned}
& \mathcal{C}_{1}=\left\{d z-p d x-q d y, d p-\frac{1}{2} \sin (2 z) d y, d x\right\} \\
& \mathcal{C}_{2}=\left\{d z-p d x-q d y, d q-\frac{1}{2} \sin (2 z) d x, d y\right\}
\end{aligned}
$$

Given two hyperbolic Monge-Ampère systems $\left(\mathcal{M}_{1}, \mathcal{I}_{1}\right)$ and $\left(\mathcal{M}_{2}, \mathcal{I}_{2}\right)$, we define a (non-parametric) Bäcklund transformation between $\left(\mathcal{M}_{1}, \mathcal{I}_{1}\right)$ and $\left(\mathcal{M}_{2}, \mathcal{I}_{2}\right)$ to be a 6-dimensional submanifold $\mathcal{B} \subset \mathcal{M}_{1} \times \mathcal{M}_{2}$ which has the following properties:

1. The natural projections $\pi_{1}: \mathcal{B} \rightarrow \mathcal{M}_{1}$ and $\pi_{2}: \mathcal{B} \rightarrow \mathcal{M}_{2}$ are submersions.

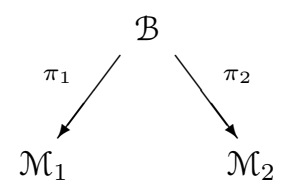

2. The pullbacks to $\mathcal{B}$ of the forms $\Theta_{1}, \Theta_{2}, \Psi_{1}, \Psi_{2}$ satisfy the condition that

$$
\left\{\Psi_{1}, \Psi_{2}\right\} \equiv\left\{\Theta_{1}, \Theta_{2}\right\} \bmod \theta_{1}, \theta_{2} .
$$

Since $\Theta_{1}, \Psi_{1}$ are linearly independent forms (as are $\Theta_{2}, \Psi_{2}$ ), this condition implies that

$$
\left\{\Theta_{1}, \Psi_{1}\right\} \equiv\left\{\Theta_{2}, \Psi_{2}\right\} \bmod \theta_{1}, \theta_{2} .
$$

This second equation is really the desired property; the first equation ensures that, in addition, the forms $\Theta_{1}, \Theta_{2}$ are linearly independent. We will let $\mathcal{J}$ denote the EDS on $\mathcal{B}$ generated by the forms $\left\{\theta_{1}, \theta_{2}, \Theta_{1}, \Theta_{2}\right\}$.

That this definition captures the properties of the sine-Gordon example may be seen as follows: suppose that $\mathcal{N} \hookrightarrow \mathcal{M}_{1}$ is a two-dimensional integral manifold of $\mathcal{I}_{1}$. 
The inverse image $\pi_{1}^{-1}(\mathcal{N})$ is a three-dimensional submanifold of $\mathcal{B}$. Now consider the restriction of $\pi_{2}^{*}\left(\mathcal{I}_{2}\right)$ to $\pi_{1}^{-1}(\mathcal{N})$. By Property 2 above, the restriction of $\pi_{2}^{*}\left(\mathcal{I}_{2}\right)$ to $\pi_{1}^{-1}(\mathcal{N})$ is a Frobenius system (i.e., a Pfaffian system which is generated algebraically by its 1 -forms). By the Frobenius Theorem (see, e.g., [1]), $\pi_{1}^{-1}(\mathcal{N})$ is foliated by two-dimensional integral manifolds of $\pi_{2}^{*}\left(\mathcal{I}_{2}\right)$, each of which projects to an integral manifold of $\left(\mathcal{M}_{2}, \mathcal{I}_{2}\right)$; moreover, these integral manifolds can be constructed by solving ODEs.

Example (cont'd). Let $\mathcal{M}_{1}=\mathcal{M}_{2}=\mathbb{R}^{5}$ with coordinates $(x, y, z, p, q)$ on $\mathcal{M}_{1}$ and $(\bar{x}, \bar{y}, \bar{z}, \bar{p}, \bar{q})$ on $\mathcal{M}_{2}$. Let $\mathcal{I}_{1}$ and $\mathcal{I}_{2}$ be copies of the sine-Gordon system $\mathcal{I}$ described above, defined on $\mathcal{M}_{1}$ and $\mathcal{M}_{2}$, respectively. For any fixed value of $\lambda$, the Bäcklund transformation (11) may be regarded as the 6-dimensional submanifold $\mathcal{B} \subset \mathcal{M}_{1} \times \mathcal{M}_{2}$ defined by the four equations

$$
\begin{gathered}
\bar{x}=x, \quad \bar{y}=y, \\
p-\bar{p}=\lambda \sin (z+\bar{z}), \\
q+\bar{q}=\frac{1}{\lambda} \sin (z-\bar{z}) .
\end{gathered}
$$

Remark. The definition given above for Bäcklund transformations can be generalized to arbitrary exterior differential systems $\mathcal{I}_{1}, \mathcal{I}_{2}$ in such a way that it defines an equivalence relation; see [1] for details.

In [6], the first author used Cartan's method of equivalence to classify all homogeneous non-parametric Bäcklund transformations of hyperbolic Monge-Ampère systems. (Here, "homogeneous" means that the $G$-structure constructed via the method of equivalence is assumed to be acted on by a transitive symmetry group, implying that all structure functions are constants.) The restriction to the homogeneous case was necessary in order to simplify the computations in the method of equivalence so that the classification could be completed explicitly. Unfortunately, homogeneity is a significant restriction. In particular, the Bäcklund transformation for the sine-Gordon equation is not homogeneous, so we expect that there are many interesting transformations which do not appear in that classification.

In the present paper, we wish to explore Bäcklund transformations that depend explicitly on an arbitrary parameter, as in the sine-Gordon case. To this end, we will provisionally define a parametric Bäcklund transformation between two hyperbolic Monge-Ampère systems $\left(\mathcal{N}_{1}, \mathcal{I}_{1}\right)$ and $\left(\mathcal{M}_{2}, \mathcal{I}_{2}\right)$ to be a 7 -dimensional submanifold

$$
\mathcal{P} \subset \mathcal{M}_{1} \times \mathcal{M}_{2} \times \mathbb{R}
$$

with coordinate $\lambda$ on the $\mathbb{R}$ factor, such that for each $\lambda_{0} \in \mathbb{R}$, the set

$$
\mathcal{B}_{\lambda_{0}}=\mathcal{P} \cap\left(\mathcal{M}_{1} \times \mathcal{M}_{2} \times\left\{\lambda_{0}\right\}\right)
$$

is a non-parametric Bäcklund transformation. (We will let $\pi_{1}, \pi_{2}$ also denote the submersions from $\mathcal{P}$ to $\mathcal{M}_{1}, \mathcal{M}_{2}$ respectively.) We will associate a canonical $G$ structure to such a transformation, and we will see that the transformation's nontrivial dependence on $\lambda$ will be equivalent to the nonvanishing of certain invariants appearing in the structure equations (see Definition 2.5 below).

Ultimately, we hope to explore the relationship between Bäcklund transformations and other features typically associated with integrable systems, such as symmetries of the system or an infinite hierarchy of conservation laws (of arbitrarily 
high order). In this paper we will explore in some detail the behavior of four examples of parametric Bäcklund transformations:

- the sine-Gordon transformation described above;

- a new parametric transformation, based on a non-parametric transformation given by Zvyagin [17, between the wave equation and a quasilinear PDE which we refer to as Goursat's equation;

- the classical auto-Bäcklund transformation between surfaces of constant Gauss curvature $K=-1$ in $\mathbb{E}^{3}$;

- a new auto-Bäcklund transformation between timelike surfaces of constant mean curvature (CMC) equal to 1 in the negatively curved Lorentzian space form $\mathbf{H}^{2,1}$ (also known as anti-de Sitter space).

We will see that:

- In the first two examples, nontrivial dependence on a parameter arises from symmetries of the underlying Monge-Ampère systems $\left(\mathcal{M}_{1}, \mathcal{I}_{1}\right),\left(\mathcal{M}_{2}, \mathcal{I}_{2}\right)$, but no such symmetries lead to the parametric dependence in the other two examples.

- In the first two examples, each characteristic system contains a rank one integrable subsystem, whereas in the $K=-1$ example none of the characteristic systems contains such a subsystem, and in the CMC example exactly one of the characteristic systems for each of $\mathcal{I}_{1}$ and $\mathcal{I}_{2}$ contains such a subsystem. For this reason we regard the CMC example as somehow exhibiting behavior "in between" that of the other examples.

- In the sine-Gordon example, each Monge-Ampère system has a 3-dimensional space of conservation laws (specifically, first-order conservation laws; see $\S 3$ for more details), while the space of conservation laws for the system $\mathcal{J}$ on $\mathcal{B}_{\lambda_{0}}$ is 4-dimensional, and is the sum of the pullbacks of the spaces of conservation laws from $\mathcal{M}_{1}$ and $\mathcal{M}_{2}$. By contrast, in the $K=-1$ example each Monge-Ampère system has a 6 -dimensional space of conservation laws, and the space of conservation laws on $\mathcal{B}_{\lambda_{0}}$ is a 7-dimensional space in which the sum of the pullbacks forms a 6-dimensional subspace. (In the other two examples the space of conservation laws for each Monge-Ampère system is infinite-dimensional.)

In the sequel to this paper, we plan to determine the relationship between these properties and certain invariants appearing in the structure equations of the $G$ structure associated to parametric Bäcklund transformations in general. Some initial results in this direction will appear in Section 7 .

\section{A canonical $G$-Structure}

In this section we will associate a canonical $G$-structure to a parametric Bäcklund transformation between two Monge-Ampère systems $\left(\mathcal{M}_{1}, \mathcal{I}_{1}\right),\left(\mathcal{M}_{2}, \mathcal{I}_{2}\right)$.

Suppose that $\mathcal{P} \subset \mathcal{M}_{1} \times \mathcal{M}_{2} \times \mathbb{R}$ is a parametric Bäcklund transformation as described above. Let $\bar{\theta}_{1}, \bar{\theta}_{2}$ denote locally defined 1 -forms on $\mathcal{P}$ which are the pullbacks of the contact forms on $\mathcal{M}_{1}, \mathcal{M}_{2}$, respectively. (For the present, we will use barred letters to denote forms and functions on $\mathcal{P}$ and unbarred letters to denote the corresponding semi-basic forms and functions defined on a $G$-structure over $\mathcal{P}$.) Let $\bar{\zeta}$ be an integrable 1 -form on $\mathcal{P}$ whose pullback to each slice $\mathcal{B}_{\lambda_{0}}$ vanishes identically, so that $\bar{\zeta}$ is a multiple of $d \lambda$, and let $\mathcal{K}$ denote the span of $\left\{\bar{\theta}_{1}, \bar{\theta}_{2}, \bar{\zeta}\right\}$. 
Recall that the Cartan system (also called the retracting space [1]) of a Pfaffian system $\mathcal{I}$ is the smallest Frobenius system containing $\mathcal{I}$. In particular, the Cartan system of a single 1 -form $\theta$ is spanned by $\theta$ and the forms which appear in $d \theta \bmod$ $\theta$, and the dimension of this Cartan system is the Pfaff rank of $\theta$. Since

$$
d \bar{\zeta} \equiv 0 \bmod \bar{\zeta}
$$

the Pfaff rank of $\bar{\zeta}$ is one. Since each $\bar{\theta}_{i}$ is the pullback of a contact form on a 5dimensional manifold, its Cartan system has rank 5 and consists of 1 -forms which are semi-basic for $\pi_{i}$, i.e., they vanish on vectors tangent to the fibers of $\pi_{i}$. We are free to modify each of $\bar{\theta}_{1}, \bar{\theta}_{2}$, and $\bar{\zeta}$ by multiplying by a nonzero function, and if we do so, their Pfaff ranks and Cartan systems do not change.

Let $\mathcal{C}_{i 1}, \mathcal{C}_{i 2}$ denote the Monge characteristic systems of $\mathcal{I}_{i}$. Since each slice $\mathcal{B}_{\lambda_{0}}$ defines a Bäcklund transformation between $\mathcal{I}_{1}$ and $\mathcal{I}_{2}$, there are 1 -forms $\bar{\omega}^{1}, \bar{\omega}^{2}, \bar{\omega}^{3}$, $\bar{\omega}^{4}$ on $\mathcal{P}$ such that

$$
\begin{array}{ll}
\pi_{1}^{*} \mathcal{C}_{11}=\left\{\bar{\theta}_{1}, \bar{\omega}^{1}, \bar{\omega}^{2}\right\}, & \pi_{2}^{*} \mathcal{C}_{21}=\left\{\bar{\theta}_{2}, \bar{\omega}^{1}, \bar{\omega}^{2}\right\}, \\
\pi_{1}^{*} \mathcal{C}_{12}=\left\{\bar{\theta}_{1}, \bar{\omega}^{3}, \bar{\omega}^{4}\right\}, & \pi_{2}^{*} \mathcal{C}_{22}=\left\{\bar{\theta}_{2}, \bar{\omega}^{3}, \bar{\omega}^{4}\right\},
\end{array}
$$

when restricted to $\mathcal{B}_{\lambda_{0}}$. (See [6] for a detailed construction of this coframe on any slice.) Since $d \bar{\theta}_{i} \bmod \bar{\theta}_{i}$ must be a linear combination of decomposables in $\mathcal{C}_{i 1}$ and $\mathcal{C}_{i 2}$, we have

$$
\begin{array}{ll}
d \bar{\theta}_{1} \equiv \bar{A}_{1} \bar{\omega}^{1} \wedge \bar{\omega}^{2}+\bar{A}_{3} \bar{\omega}^{3} \wedge \bar{\omega}^{4} \quad \bmod \bar{\theta}_{1}, \bar{\zeta}, \\
d \bar{\theta}_{2} \equiv \bar{A}_{4} \bar{\omega}^{1} \wedge \bar{\omega}^{2}+\bar{A}_{2} \bar{\omega}^{3} \wedge \bar{\omega}^{4} \quad \bmod \bar{\theta}_{2}, \bar{\zeta}
\end{array}
$$

for some nonzero functions $\bar{A}_{i}$ on $\mathcal{P}$ such that $\bar{A}_{1} \bar{A}_{2}-\bar{A}_{3} \bar{A}_{4} \neq 0$ at each point. This implies the analogue of (44),

$$
\left\{\pi_{1}^{*} \bar{\Psi}_{1}, \pi_{2}^{*} \bar{\Psi}_{2}\right\} \equiv\left\{d \bar{\theta}_{1}, d \bar{\theta}_{2}\right\} \quad \bmod \mathcal{K} .
$$

We are free to modify the $\bar{\omega}^{i}$ by scaling and adding multiples of $\bar{\zeta}$, and we will use this to arrange that $\bar{A}_{3}=1=\bar{A}_{4}$ and that, on $\mathcal{P}$,

$$
\pi_{1}^{*} \mathcal{C}_{11}=\left\{\bar{\theta}_{1}, \bar{\omega}^{1}, \bar{\omega}^{2}\right\}, \quad \pi_{2}^{*} \mathcal{C}_{22}=\left\{\bar{\theta}_{2}, \bar{\omega}^{3}, \bar{\omega}^{4}\right\} .
$$

(This condition determines the spans $\left\{\bar{\omega}^{1}, \bar{\omega}^{2}\right\}$ and $\left\{\bar{\omega}^{3}, \bar{\omega}^{4}\right\}$ uniquely.) Consequently, there are functions $\bar{D}_{i}$ on $\mathcal{P}$ such that

$$
\begin{array}{ll}
d \bar{\theta}_{1} \equiv \bar{A}_{1} \bar{\omega}^{1} \wedge \bar{\omega}^{2}+\bar{\omega}^{3} \wedge \bar{\omega}^{4}+\bar{\zeta} \wedge\left(\bar{D}_{3} \bar{\omega}^{3}+\bar{D}_{4} \bar{\omega}^{4}\right) & \bmod \bar{\theta}_{1}, \\
d \bar{\theta}_{2} \equiv \bar{\omega}^{1} \wedge \bar{\omega}^{2}+\bar{A}_{2} \bar{\omega}^{3} \wedge \bar{\omega}^{4}+\bar{\zeta} \wedge\left(\bar{D}_{1} \bar{\omega}^{1}+\bar{D}_{2} \bar{\omega}^{2}\right) & \bmod \bar{\theta}_{2}
\end{array}
$$

with $\bar{A}_{1} \bar{A}_{2} \neq 1$, and

(9) $\pi_{1}^{*} \mathcal{C}_{12}=\left\{\bar{\theta}_{1}, \bar{\omega}^{3}+\bar{D}_{4} \bar{\zeta}, \bar{\omega}^{4}-\bar{D}_{3} \bar{\zeta}\right\}, \quad \pi_{2}^{*} \mathcal{C}_{21}=\left\{\bar{\theta}_{2}, \bar{\omega}^{1}+\bar{D}_{2} \bar{\zeta}, \bar{\omega}^{2}-\bar{D}_{1} \bar{\zeta}\right\}$.

Now let $\mathcal{G}$ be the sub-bundle of the coframe bundle of $\mathcal{P}$ consisting of all coframes $\left(\bar{\zeta}, \bar{\theta}_{1}, \bar{\theta}_{2}, \bar{\omega}^{1}, \bar{\omega}^{2}, \bar{\omega}^{3}, \bar{\omega}^{4}\right)$ satisfying the conditions (5), (7), (8), (9) above. Then $\mathcal{G}$ is a $G$-structure on $\mathcal{P}$, where $G \subset G L(7, \mathbb{R})$ is the 9-dimensional group of matrices of the form

$$
\left\{\left[\begin{array}{ccccc}
t & 0 & 0 & & \\
0 & s & 0 & 0 & 0 \\
0 & 0 & r & r & 0 \\
0 & R & 0 \\
0 & 0 & S
\end{array}\right] \mid R, S \in G L(2, \mathbb{R}), r=\operatorname{det}(R), s=\operatorname{det}(S), t \neq 0\right\} .
$$


There are canonical 1 -forms $\left(\zeta, \theta_{1}, \theta_{2}, \omega^{1}, \omega^{2}, \omega^{3}, \omega^{4}\right)$ on $\mathcal{G}$ defined by the property that for any section $\sigma=\left(\bar{\zeta}, \bar{\theta}_{1}, \bar{\theta}_{2}, \bar{\omega}^{1}, \bar{\omega}^{2}, \bar{\omega}^{3}, \bar{\omega}^{4}\right): \mathcal{P} \rightarrow \mathcal{G}$ we have

$$
\sigma^{*}(\zeta)=\bar{\zeta}, \sigma^{*}\left(\theta_{i}\right)=\bar{\theta}_{i}, \sigma^{*}\left(\omega^{i}\right)=\bar{\omega}^{i} .
$$

These forms are semi-basic for the projection from $\mathcal{G}$ to $\mathcal{P}$ and will henceforth be referred to as the semi-basic forms on $\mathcal{G}$. The reproducing property (10) implies that

$$
\begin{array}{ll}
d \theta_{1} \equiv A_{1} \omega^{1} \wedge \omega^{2}+\omega^{3} \wedge \omega^{4}+\zeta \wedge\left(D_{3} \omega^{3}+D_{4} \omega^{4}\right) & \bmod \theta_{1}, \\
d \theta_{2} \equiv \omega^{1} \wedge \omega^{2}+A_{2} \omega^{3} \wedge \omega^{4}+\zeta \wedge\left(D_{1} \omega^{1}+D_{2} \omega^{2}\right) & \bmod \theta_{2}
\end{array}
$$

where $A_{i}, D_{i}$ are functions on $\mathcal{G}$ such that $\sigma^{*}\left(A_{i}\right)=\bar{A}_{i}$ and $\sigma^{*}\left(D_{i}\right)=\bar{D}_{i}$ for any section $\sigma$. The Cartan systems $\mathcal{R}_{1}, \mathcal{R}_{2}$ of $\theta_{1}, \theta_{2}$ on $\mathcal{G}$ are given by

$$
\mathcal{R}_{1}=\left\{\theta_{1}, \omega^{1}, \omega^{2}, \widetilde{\omega}^{3}, \widetilde{\omega}^{4}\right\}, \quad \mathcal{R}_{2}=\left\{\theta_{2}, \widetilde{\omega}^{1}, \widetilde{\omega}^{2}, \omega^{3}, \omega^{4}\right\},
$$

where, for the sake of convenience, we define

$$
\widetilde{\omega}^{1}=\omega^{1}+D_{2} \zeta, \quad \widetilde{\omega}^{2}=\omega^{2}-D_{1} \zeta, \quad \widetilde{\omega}^{3}=\omega^{3}+D_{4} \zeta, \quad \widetilde{\omega}^{4}=\omega^{4}-D_{3} \zeta .
$$

We will now proceed with the method of equivalence by trying to normalize terms appearing in the exterior derivatives of the semi-basic forms on $\mathcal{G}$. First, we must compute the exterior derivatives of the $\omega^{i}$.

Note that the pullbacks of the Monge characteristic systems of $\mathcal{I}_{1}$ are given by $\left\{\theta_{1}, \omega^{1}, \omega^{2}\right\}$ and $\left\{\theta_{1}, \widetilde{\omega}^{3}, \widetilde{\omega}^{4}\right\}$, while the pullbacks of those of $\mathcal{I}_{2}$ are $\left\{\theta_{2}, \widetilde{\omega}^{1}, \widetilde{\omega}^{2}\right\}$ and $\left\{\theta_{2}, \omega^{3}, \omega^{4}\right\}$. This implies that

$$
\begin{array}{ll}
d \omega^{1} \equiv d \omega^{2} \equiv 0 & \bmod \theta_{1}, \omega^{1}, \omega^{2}, \Lambda^{2}\left(\mathcal{R}_{1}\right), \\
d \omega^{3} \equiv d \omega^{4} \equiv 0 & \bmod \theta_{2}, \omega^{3}, \omega^{4}, \Lambda^{2}\left(\mathcal{R}_{2}\right)
\end{array}
$$

and

$$
\begin{array}{ll}
d \widetilde{\omega}^{1} \equiv d \widetilde{\omega}^{2} \equiv 0 & \bmod \theta_{2}, \widetilde{\omega}^{1}, \widetilde{\omega}^{2}, \Lambda^{2}\left(\mathcal{R}_{2}\right), \\
d \widetilde{\omega}^{3} \equiv d \widetilde{\omega}^{4} \equiv 0 & \bmod \theta_{1}, \widetilde{\omega}^{3}, \widetilde{\omega}^{4}, \Lambda^{2}\left(\mathcal{R}_{1}\right),
\end{array}
$$

where $\Lambda^{2}\left(\mathcal{R}_{i}\right)$ denotes the span of wedge products of degree two, both of whose factors belong to $\mathcal{R}_{i}$. Because $\zeta$ is integrable, (13) implies that

$$
\begin{array}{ll}
d \omega^{1} \equiv d \omega^{2} \equiv 0 & \bmod \zeta, \theta_{2}, \omega^{1}, \omega^{2}, \Lambda^{2}\left(\mathcal{R}_{2}\right), \\
d \omega^{3} \equiv d \omega^{4} \equiv 0 & \bmod \zeta, \theta_{1}, \omega^{3}, \omega^{4}, \Lambda^{2}\left(\mathcal{R}_{1}\right) .
\end{array}
$$

Together, (12) and (14) imply that

$$
\left.\begin{array}{l}
d \omega^{1} \equiv B_{1} \theta_{1} \wedge \theta_{2}+C_{1} \widetilde{\omega}^{3} \wedge \widetilde{\omega}^{4}+E_{1} \zeta \wedge \theta_{1} \\
d \omega^{2} \equiv B_{2} \theta_{1} \wedge \theta_{2}+C_{2} \widetilde{\omega}^{3} \wedge \widetilde{\omega}^{4}+E_{2} \zeta \wedge \theta_{1}
\end{array}\right\} \quad \bmod \omega^{1}, \omega^{2},
$$

for some functions $B_{i}, C_{i}$ and $E_{i}$ on $\mathcal{G}$.

A standard argument shows that on $\mathcal{G}$ there exist 1-forms $\alpha_{i}, \beta_{i}, \gamma$ (referred to as pseudo-connection, or simply connection forms), linearly independent from the 
semi-basic forms, such that

$$
d\left[\begin{array}{c}
\zeta \\
\theta_{1} \\
\theta_{2} \\
\omega^{1} \\
\omega^{2} \\
\omega^{3} \\
\omega^{4}
\end{array}\right]=-\left[\begin{array}{ccccccc}
\gamma & 0 & 0 & 0 & 0 & 0 & 0 \\
0 & \beta_{1}+\beta_{4} & 0 & 0 & 0 & 0 & 0 \\
0 & 0 & \alpha_{1}+\alpha_{4} & 0 & 0 & 0 & 0 \\
0 & 0 & 0 & \alpha_{1} & \alpha_{2} & 0 & 0 \\
0 & 0 & 0 & \alpha_{3} & \alpha_{4} & 0 & 0 \\
0 & 0 & 0 & 0 & 0 & \beta_{1} & \beta_{2} \\
0 & 0 & 0 & 0 & 0 & \beta_{3} & \beta_{4}
\end{array}\right] \wedge\left[\begin{array}{c}
\zeta \\
\theta_{1} \\
\theta_{2} \\
\omega^{1} \\
\omega^{2} \\
\omega^{3} \\
\omega^{4}
\end{array}\right]+\left[\begin{array}{c}
\Upsilon \\
\Theta_{1} \\
\Theta_{2} \\
\Omega^{1} \\
\Omega^{2} \\
\Omega^{3} \\
\Omega^{4}
\end{array}\right] .
$$

These are the structure equations of $\mathcal{G}$. The column on the far right consists of 2 -forms which are sums of wedge products of pairs of semi-basic forms on 9 ; these forms are collectively known as torsion. Our assumptions about the $G$-structure limit the kinds of terms that can occur as torsion, and we may also absorb some torsion terms by adding semi-basic forms to the connection forms. In particular, we can arrange that

$$
\begin{aligned}
\Upsilon & =0 \\
\Theta_{1} & =F_{1} \zeta \wedge \theta_{1}+A_{1}\left(\omega^{1}-C_{1} \theta_{1}\right) \wedge\left(\omega^{2}-C_{2} \theta_{1}\right)+\left(\omega^{3}+D_{4} \zeta\right) \wedge\left(\omega^{4}-D_{3} \zeta\right), \\
\Theta_{2} & =F_{2} \zeta \wedge \theta_{2}+A_{2}\left(\omega^{3}-C_{3} \theta_{2}\right) \wedge\left(\omega^{4}-C_{4} \theta_{2}\right)+\left(\omega^{1}+D_{2} \zeta\right) \wedge\left(\omega^{2}-D_{1} \zeta\right), \\
\Omega_{1} & =B_{1} \theta_{1} \wedge \theta_{2}+C_{1}\left(\omega^{3}+D_{4} \zeta\right) \wedge\left(\omega^{4}-D_{3} \zeta\right)+E_{1} \zeta \wedge \theta_{1}, \\
\Omega_{2} & =B_{2} \theta_{1} \wedge \theta_{2}+C_{2}\left(\omega^{3}+D_{4} \zeta\right) \wedge\left(\omega^{4}-D_{3} \zeta\right)+E_{2} \zeta \wedge \theta_{1}, \\
\Omega_{3} & =B_{3} \theta_{1} \wedge \theta_{2}+C_{3}\left(\omega^{1}+D_{2} \zeta\right) \wedge\left(\omega^{2}-D_{1} \zeta\right)+E_{3} \zeta \wedge \theta_{2}, \\
\Omega_{4} & =B_{4} \theta_{1} \wedge \theta_{2}+C_{4}\left(\omega^{1}+D_{2} \zeta\right) \wedge\left(\omega^{2}-D_{1} \zeta\right)+E_{4} \zeta \wedge \theta_{2}
\end{aligned}
$$

for some functions $A_{i}, B_{i}, C_{i}, D_{i}, E_{i}, F_{i}$ on $\mathcal{G}$. (As noted previously, we must have $A_{1}, A_{2}$ nonzero and $A_{1} A_{2} \neq 1$.)

The right action of $G$ on sections $\sigma$ of $\mathcal{G}$ is $\sigma \cdot g=g^{-1} \sigma$. This induces an action on the torsion coefficients, as follows. The functions $A_{1}, A_{2}, F_{1}, F_{2}$ are acted on by scaling:

$$
A_{1} \rightarrow r s^{-1} A_{1}, \quad A_{2} \rightarrow r^{-1} s A_{2}, \quad F_{1} \rightarrow t F_{1}, \quad F_{2} \rightarrow t F_{2} .
$$

(In particular, the product $A_{1} A_{2}$ is invariant on the fibers of $\mathcal{G}$, so the condition $A_{1} A_{2} \neq 1$ makes sense.) The remaining coefficients occur naturally in pairs as components of vectors and are acted on as follows:

$$
\begin{array}{ll}
{\left[\begin{array}{l}
B_{1} \\
B_{2}
\end{array}\right] \rightarrow r s R^{-1}\left[\begin{array}{l}
B_{1} \\
B_{2}
\end{array}\right]} & {\left[\begin{array}{l}
B_{3} \\
B_{4}
\end{array}\right] \rightarrow r s S^{-1}\left[\begin{array}{l}
B_{3} \\
B_{4}
\end{array}\right],} \\
{\left[\begin{array}{l}
C_{1} \\
C_{2}
\end{array}\right] \rightarrow s R^{-1}\left[\begin{array}{l}
C_{1} \\
C_{2}
\end{array}\right]} & {\left[\begin{array}{l}
C_{3} \\
C_{4}
\end{array}\right] \rightarrow r S^{-1}\left[\begin{array}{l}
C_{3} \\
C_{4}
\end{array}\right],} \\
{\left[\begin{array}{l}
D_{1} \\
D_{2}
\end{array}\right] \rightarrow\left(r^{-1} t\right)^{t} R\left[\begin{array}{l}
D_{1} \\
D_{2}
\end{array}\right]} & {\left[\begin{array}{l}
D_{3} \\
D_{4}
\end{array}\right] \rightarrow\left(s^{-1} t\right)^{t} S\left[\begin{array}{l}
D_{3} \\
D_{4}
\end{array}\right],} \\
{\left[\begin{array}{l}
E_{1} \\
E_{2}
\end{array}\right] \rightarrow s t R^{-1}\left[\begin{array}{l}
E_{1} \\
E_{2}
\end{array}\right]} & {\left[\begin{array}{l}
E_{3} \\
E_{4}
\end{array}\right] \rightarrow r t S^{-1}\left[\begin{array}{l}
E_{3} \\
E_{4}
\end{array}\right] .}
\end{array}
$$


In particular, we observe that the vanishing of any of these vectors is a well-defined condition on $\mathcal{P}$, as is the linear dependence or independence of certain pairs of vectors, e.g., $\left[\begin{array}{ll}B_{1} & B_{2}\end{array}\right]$ and $\left[\begin{array}{ll}C_{1} & C_{2}\end{array}\right]$ or $\left[\begin{array}{ll}E_{1} & E_{2}\end{array}\right]$. As well, the dot product of $\left[\begin{array}{ll}D_{1} & D_{2}\end{array}\right]$ with any one of these three is a relative invariant, i.e., a quantity upon which $G$ acts by a nonzero scale factor.

We can make some elementary observations regarding how the vanishing of some of these vectorial invariants implies the vanishing of others.

Proposition 2.1. If the vector $\left[C_{1} C_{2}\right]$ vanishes identically on $\mathcal{P}$, then so do $\left[B_{1} B_{2}\right]$ and $\left[\begin{array}{ll}E_{1} & E_{2}\end{array}\right]$. Similarly, if $\left[C_{3} C_{4}\right]$ vanishes, then so do $\left[\begin{array}{ll}B_{3} & B_{4}\end{array}\right]$ and $\left[\begin{array}{ll}E_{3} & E_{4}\end{array}\right]$.

Proof. Suppose that $C_{1}=C_{2}=0$. Differentiating the structure equations for $d \omega^{1}$ and $d \omega^{2}$ and reducing modulo $\theta_{1}, \omega^{1}$, and $\omega^{2}$ yields

$$
\begin{aligned}
& 0=d\left(d \omega^{1}\right) \equiv\left(B_{1} \theta_{2}-E_{1} \zeta\right) \wedge \widetilde{\omega}^{3} \wedge \widetilde{\omega}^{4} \bmod \theta_{1}, \omega^{1}, \omega^{2}, \\
& 0=d\left(d \omega^{2}\right) \equiv\left(B_{2} \theta_{2}-E_{2} \zeta\right) \wedge \widetilde{\omega}^{3} \wedge \widetilde{\omega}^{4} \bmod \theta_{1}, \omega^{1}, \omega^{2} ;
\end{aligned}
$$

therefore, $B_{1}=B_{2}=E_{1}=E_{2}=0$. A similar argument shows that if $\left[C_{3} C_{4}\right]$ vanishes, then so do $\left[\begin{array}{ll}B_{3} & B_{4}\end{array}\right]$ and $\left[\begin{array}{ll}E_{3} & E_{4}\end{array}\right]$.

In all the examples considered below, both vectors $\left[\begin{array}{ll}C_{1} & C_{2}\end{array}\right]$ and $\left[\begin{array}{ll}C_{3} & C_{4}\end{array}\right]$ are nonzero.

The following two propositions are proved in [6].

Proposition 2.2. If both vectors $\left[C_{1} C_{2}\right],\left[C_{3} C_{4}\right]$ vanish, then the Bäcklund transformation is locally equivalent to a transformation between solutions of the wave equation $z_{x y}=0$.

Proposition 2.3. If both of the vectors $\left[\begin{array}{ll}C_{1} & C_{2}\end{array}\right],\left[\begin{array}{ll}C_{3} & C_{4}\end{array}\right]$ are nonzero, then the vectors $\left[\begin{array}{ll}B_{1} & B_{2}\end{array}\right],\left[\begin{array}{ll}B_{3} & B_{4}\end{array}\right]$ are either both zero or both nonzero.

If the vectors $\left[\begin{array}{ll}B_{1} & B_{2}\end{array}\right]$ and $\left[\begin{array}{ll}B_{3} & B_{4}\end{array}\right]$ both vanish, then for each $\lambda$ the Bäcklund transformation is holonomic in the sense described in [6].

Proposition 2.4. If both of the vectors $\left[C_{1} C_{2}\right],\left[\begin{array}{ll}C_{3} & C_{4}\end{array}\right]$ are nonzero, then the vectors $\left[\begin{array}{ll}D_{1} & D_{2}\end{array}\right],\left[\begin{array}{ll}D_{3} & D_{4}\end{array}\right]$ are either both zero or both nonzero. Moreover, if the $D$-vectors both vanish, then so do the vectors $\left[\begin{array}{ll}E_{1} & E_{2}\end{array}\right],\left[\begin{array}{ll}E_{3} & E_{4}\end{array}\right]$ and the functions $F_{1}, F_{2}$.

Proof. Suppose that $D_{1}=D_{2}=0$. Differentiating the structure equation for $d \theta_{2}$ and reducing modulo $\theta_{1}, \theta_{2}$, either $\omega^{1}$ or $\omega^{2}$, and either $\omega^{3}$ or $\omega^{4}$ yields

$$
\begin{aligned}
& 0=d\left(d \theta_{2}\right) \equiv-C_{1} D_{4} \zeta \wedge \omega^{2} \wedge \omega^{4} \bmod \theta_{1}, \theta_{2}, \omega^{1}, \omega^{3}, \\
& 0=d\left(d \theta_{2}\right) \equiv C_{2} D_{4} \zeta \wedge \omega^{1} \wedge \omega^{4} \bmod \theta_{1}, \theta_{2}, \omega^{2}, \omega^{3}, \\
& 0=d\left(d \theta_{2}\right) \equiv-C_{1} D_{3} \zeta \wedge \omega^{2} \wedge \omega^{3} \bmod \theta_{1}, \theta_{2}, \omega^{1}, \omega^{4}, \\
& 0=d\left(d \theta_{2}\right) \equiv C_{2} D_{3} \zeta \wedge \omega^{1} \wedge \omega^{3} \bmod \theta_{1}, \theta_{2}, \omega^{2}, \omega^{4} .
\end{aligned}
$$

Since $C_{1}$ and $C_{2}$ are not both zero, we must have $D_{3}=D_{4}=0$. A similar argument shows the converse.

Now suppose that $D_{1}=D_{2}=D_{3}=D_{4}=0$. Differentiating the structure equation for $d \theta_{1}$ yields

$$
0=d\left(d \theta_{1}\right) \equiv \zeta \wedge\left(E_{3} \theta_{2} \wedge \omega^{4}-E_{4} \theta_{2} \wedge \omega^{3}-F_{1} \omega^{3} \wedge \omega^{4}\right) \quad \bmod \theta_{1}, \omega^{1}, \omega^{2} ;
$$


therefore, $E_{3}=E_{4}=F_{1}=0$. Similarly, differentiating the structure equation for $d \theta_{2}$ yields

$$
0=d\left(d \theta_{2}\right) \equiv \zeta \wedge\left(E_{1} \theta_{1} \wedge \omega^{2}-E_{2} \theta_{1} \wedge \omega^{1}-F_{2} \omega^{1} \wedge \omega^{2}\right) \quad \bmod \theta_{2}, \omega^{3}, \omega^{4} ;
$$

therefore, $E_{1}=E_{2}=F_{2}=0$.

From this proposition we see that if either of the vectors $\left[\begin{array}{ll}D_{1} & D_{2}\end{array}\right],\left[\begin{array}{ll}D_{3} & D_{4}\end{array}\right]$ vanishes (and the corresponding $C$-vector is nonzero), then the structure equations for $d \theta_{i}, d \omega^{i}$ contain no terms involving $\zeta$. In that case, the Bäcklund transformation depends trivially on the parameter $\lambda$. Conversely, if these vectors are nonzero, then the Bäcklund transformation has nontrivial dependence upon $\lambda$ and may be considered genuinely parametric. For this reason, we make the following definition.

Definition 2.5. A parametric Bäcklund transformation between two hyperbolic Monge-Ampère systems $\left(\mathcal{M}_{1}, \mathcal{I}_{1}\right)$ and $\left(\mathcal{M}_{2}, \mathcal{I}_{2}\right)$ is a 7 -dimensional submanifold

$$
\mathcal{P} \subset \mathcal{M}_{1} \times \mathcal{M}_{2} \times \mathbb{R}
$$

as described in the introduction, with the additional condition that the vectors $\left[\begin{array}{ll}D_{1} & D_{2}\end{array}\right]$ and $\left[\begin{array}{ll}D_{3} & D_{4}\end{array}\right]$ do not both vanish.

\section{The Sine-Gordon Equation}

In this and subsequent sections, we will drop the usage of bars to distinguish between objects on $\mathcal{P}$ and the corresponding objects on $\mathcal{G}$. (We will be working mostly on $\mathcal{P}$ anyway.) Instead, bars will be used to distinguish between similar coordinates (or similar coframes) on $\mathcal{M}_{1}$ and $\mathcal{M}_{2}$.

Bäcklund transformation. As in the example given in the introduction, let $\mathcal{M}_{1}=$ $\mathcal{M}_{2}=\mathbb{R}^{5}$ with coordinates $(x, y, z, p, q)$ on $\mathcal{M}_{1}$ and $(\bar{x}, \bar{y}, \bar{z}, \bar{p}, \bar{q})$ on $\mathcal{M}_{2}$. Let $\mathcal{I}_{1}$ and $\mathcal{I}_{2}$ be copies of the sine-Gordon system generated by the forms

$$
\begin{aligned}
\theta_{1} & =d z-p d x-q d y \\
\Theta_{1} & =-d p \wedge d x-d q \wedge d y \\
\Psi_{1}=\left(d p-\frac{1}{2} \sin (2 z) d y\right) & \wedge d x \equiv-\left(d q-\frac{1}{2} \sin (2 z) d x\right) \wedge d y \quad \bmod \Theta_{1}
\end{aligned}
$$

on $\mathcal{M}_{1}$, and similar forms $\theta_{2}, \Theta_{2}, \Psi_{2}$ in terms of the barred variables on $\mathcal{M}_{2}$. The Bäcklund transformation $\mathcal{P} \subset \mathcal{M}_{1} \times \mathcal{M}_{2} \times \mathbb{R}$ is defined by the four equations

$$
\begin{gathered}
\bar{x}=x, \quad \bar{y}=y, \\
p-\bar{p}=\lambda \sin (z+\bar{z}), \\
q+\bar{q}=\frac{1}{\lambda} \sin (z-\bar{z}) .
\end{gathered}
$$

Let $\mathcal{K}$ denote the span of the 1 -forms $\theta_{1}, \theta_{2}$ and $d \lambda$. On $\mathcal{P}$, we have

$$
\begin{array}{rlrl}
d \bar{p}-\frac{1}{2} \sin (2 \bar{z}) d y & \equiv d p-\frac{1}{2} \sin (2 z) d y & \bmod d x, \mathcal{K}, \\
d \bar{q}-\frac{1}{2} \sin (2 \bar{z}) d x & \equiv-\left(d q-\frac{1}{2} \sin (2 z) d x\right) & & \bmod d y, \mathcal{K} .
\end{array}
$$

From these, it follows that $\Theta_{1} \equiv-2 \Psi_{2}$ and $\Theta_{2} \equiv-2 \Psi_{1}$ modulo $\mathcal{K}$. 
$G$-structure invariants. We will use $x, y, z, \bar{z}, \bar{p}, q$, and $\lambda$ as coordinates on $\mathcal{P}$; then $p, \bar{q}$ are given by the Bäcklund transformation equations

$$
\begin{aligned}
& p=\bar{p}+\lambda \sin (z+\bar{z}), \\
& \bar{q}=-q+\frac{1}{\lambda} \sin (z-\bar{z}) .
\end{aligned}
$$

The coframe

$$
\begin{array}{rlrl}
\zeta & =\lambda^{-1} d \lambda, & \omega^{1}=d x, \\
\theta_{1}=d z-p d x-q d y, & \omega^{2}=d \bar{p}-\frac{1}{2} \sin (2 \bar{z}) d y \\
\theta_{2}=d \bar{z}-\bar{p} d x-\bar{q} d y, & \omega^{3}=d y \\
& \omega^{4}=d q-\frac{1}{2} \sin (2 z) d x
\end{array}
$$

satisfies

$$
\begin{aligned}
& d \theta_{1} \equiv A_{1} \omega^{1} \wedge \omega^{2}+\omega^{3} \wedge \omega^{4} \\
& d \theta_{2} \equiv \omega^{1} \wedge \omega^{2}+A_{2} \omega^{3} \wedge \omega^{4}
\end{aligned} \quad \bmod \mathcal{K}
$$

with $A_{1}=1, A_{2}=-1$. To get a section of $\mathcal{G}$ we can modify $\omega^{2}$ and $\omega^{4}$ to

$$
\begin{aligned}
\omega^{2} & =d \bar{p}-\frac{1}{2} \sin (2 \bar{z}) d y+\lambda\left(\sin (z+\bar{z}) \zeta+\cos (z+\bar{z}) \theta_{2}\right) \\
\omega^{4} & =d q-\frac{1}{2} \sin (2 z) d x+\lambda^{-1}\left(\sin (z-\bar{z}) \zeta-\cos (z-\bar{z}) \theta_{1}\right) .
\end{aligned}
$$

Using this modified coframe, we compute that

$$
\begin{aligned}
{\left[\begin{array}{lll}
B_{1} & C_{1} & E_{1} \\
B_{2} & C_{2} & E_{2}
\end{array}\right] } & =-\lambda\left[\begin{array}{ccc}
0 & 0 & 0 \\
\sin (z+\bar{z}) & \cos (z+\bar{z}) & \cos (z+\bar{z})
\end{array}\right], \\
{\left[\begin{array}{l}
D_{1} \\
D_{2}
\end{array}\right] } & =\lambda\left[\begin{array}{c}
\sin (z+\bar{z}) \\
0
\end{array}\right], \\
{\left[\begin{array}{lll}
B_{3} & C_{3} & E_{3} \\
B_{4} & C_{4} & E_{4}
\end{array}\right] } & =\frac{1}{\lambda}\left[\begin{array}{ccc}
0 & 0 & 0 \\
\sin (z-\bar{z}) & -\cos (z-\bar{z}) & \cos (z-\bar{z})
\end{array}\right], \\
{\left[\begin{array}{c}
D_{3} \\
D_{4}
\end{array}\right] } & =\lambda^{-1}\left[\begin{array}{c}
\sin (\bar{z}-z) \\
0
\end{array}\right] .
\end{aligned}
$$

As well, $F_{1}=F_{2}=0$. We observe that

(1) The vector triples $\left\{\left[\begin{array}{ll}B_{1} & B_{2}\end{array}\right],\left[\begin{array}{ll}C_{1} & C_{2}\end{array}\right],\left[\begin{array}{ll}E_{1} & E_{2}\end{array}\right]\right\}$ and $\left\{\left[\begin{array}{ll}B_{3} & B_{4}\end{array}\right],\left[\begin{array}{ll}C_{3} & C_{4}\end{array}\right]\right.$, $\left.\left[\begin{array}{ll}E_{3} & E_{4}\end{array}\right]\right\}$ each span a one-dimensional space.

(2) Each $D$-vector is perpendicular to the corresponding $C$-vector.

(3) For both $\mathcal{I}_{1}$ and $\mathcal{I}_{2}$, each characteristic system contains a rank one integrable subsystem, namely, those spanned by $\{d x\}$ and $\{d y\}$.

In Section 7 we will show that condition (3) follows from conditions (1) and (2).

Symmetries. The sine-Gordon equation has a three-dimensional group of symmetries, generated by translations in $x$ and $y$ and the Lie transformation $L_{\mu}$, defined by

$$
x \mapsto \mu^{-1} x, \quad y \mapsto \mu y, \quad z \mapsto z, \quad \mu \neq 0 .
$$

This is a symmetry of the sine-Gordon equation (2), as well as any "f-Gordon" equation $z_{x y}=f(z)$. In other words, $L_{\mu}$ takes the graph of one solution $z$ to that 
of another solution $z^{\prime}=L_{\mu} \cdot z$, defined by

$$
z^{\prime}(x, y)=z\left(\mu x, \mu^{-1} y\right) .
$$

Now, suppose $z, \bar{z}$ are two solutions that satisfy the Bäcklund transformation equations (1) for $\lambda=\lambda_{0}$. Let the Lie transformation act simultaneously on both, obtaining new solutions $z^{\prime}, \bar{z}^{\prime}$ respectively. Then $z^{\prime}, \bar{z}^{\prime}$ satisfy (1) for $\lambda=\mu \lambda_{0}$. In other words, the parameter in (1) is generated by lifting the Lie transformation.

This observation is not new; in fact, Rogers and Shadwick [16] show that, assuming that the Bäcklund transformation does not change the independent variables, and that the symmetries of each differential equation are point transformations which cover the same transformation of the independent variables (e.g., $x \mapsto \mu^{-1} x$, $y \mapsto \mu y$ ), then this procedure always produces a one-parameter family of Bäcklund transformations from a single Bäcklund transformation. While it would be interesting to generalize this result to a less restrictive setting, at present we are concerned with how simultaneous symmetries in each of the Monge-Ampère equations manifest themselves as diffeomorphisms of the 7 -dimensional manifold $\mathcal{P}$. Furthermore, we will focus on infinitesimal symmetries, i.e., the vector fields which generate symmetry transformations.

A vector field $v$ is an (infinitesimal) symmetry of an $\operatorname{EDS} \mathcal{I}$ if $\mathcal{L}_{\mathrm{v}} \psi \in \mathcal{I}$ for any form $\psi \in \mathcal{I}$. (Here $\mathcal{L}$ denotes the Lie derivative.) Such vector fields form a Lie algebra $\mathfrak{S}_{\mathcal{I}}$ under the usual bracket. A special subalgebra $\mathfrak{C}_{\mathcal{I}}$ consists of Cauchy characteristic vector fields, which are simply vector fields annihilated by the 1-forms in the Cartan system of $\mathcal{I}$.

It is straightforward to check that

$$
\mathbf{v}=x \frac{\partial}{\partial x}-y \frac{\partial}{\partial y}-p \frac{\partial}{\partial p}+q \frac{\partial}{\partial q}
$$

is the infinitesimal symmetry of the Monge-Ampère system (1) generating the Lie transformation. Let $\bar{v}$ denote the corresponding vector field on $\mathcal{M}_{2}$.

Proposition 3.1. In terms of coordinates $x, y, p, q, z, \bar{z}$ and $t=\log \lambda$ on $\mathcal{P}$,

$$
\mathbf{w}=x \frac{\partial}{\partial x}-y \frac{\partial}{\partial y}-p \frac{\partial}{\partial p}+q \frac{\partial}{\partial q}-\frac{\partial}{\partial t}
$$

is the unique vector field on $\mathcal{P}$ which covers $\vee$ and $\overline{\mathrm{v}}$, i.e., $\pi_{1 *} \mathrm{w}=\mathrm{v}$ and $\pi_{2 *} \mathrm{w}=\overline{\mathrm{v}}$.

Proof. Suppose that

$$
\mathbf{w}=x \frac{\partial}{\partial x}-y \frac{\partial}{\partial y}-p \frac{\partial}{\partial p}+q \frac{\partial}{\partial q}+A \frac{\partial}{\partial \bar{z}}+B \frac{\partial}{\partial t}
$$

covers $\vee, \bar{v}$. Since $\bar{v}\lrcorner d \bar{z}=0$, we have $A=0$. Since $\bar{v}\lrcorner d \bar{p}=-\bar{p}$, we must have

$$
\begin{aligned}
-\bar{p} & =\mathrm{w}\lrcorner d \bar{p} \\
& =\mathrm{w}\lrcorner[d p-\lambda \cos (z+\bar{z})(d z+d \bar{z})-\lambda \sin (z+\bar{z}) d t] \\
& =-p-\lambda \sin (z+\bar{z}) B .
\end{aligned}
$$

Then (17) gives $B=-1$.

Note that Proposition 3.1 implies that there is no vector field tangent to the 6dimensional level sets $\mathcal{B}_{\lambda}$ of $\lambda$ which covers $v$ and $\bar{v}$. However, as has been observed 
by Igonin and Krasil'shchik [10, there is a lift of $\vee$ to $\mathcal{B}_{1}$ which generates the oneparameter family of Bäcklund transformations. We will now make this construction explicit.

Let $\hat{\mathrm{v}}$ be the lift of $\mathrm{v}$ to $\mathcal{B}=\mathcal{B}_{1}$ such that $\hat{\mathrm{v}}-d \bar{z}=0$. Then $\hat{\mathrm{v}}$ is not a symmetry of the form $\theta_{2}$, since $\mathcal{L}_{\hat{\mathrm{v}}} \theta_{2}=\sin (z+\bar{z}) d x+\sin (z-\bar{z}) d y$. Thus, flow by $\hat{v}$ induces a 1-parameter group of diffeomorphisms $\varphi_{t}$ of $\mathcal{B}$ which preserve $\theta_{1}$ up to multiple (i.e., $\varphi_{t}^{*} \theta_{1} \equiv 0 \bmod \theta_{1}$ ) but do not preserve $\theta_{2}$. These are

$$
\varphi_{t}(x, y, p, q, z, \bar{z})=\left(e^{t} x, e^{-t} y, e^{-t} p, e^{t} q, z, \bar{z}\right),
$$

Now let $\varphi: \mathcal{B} \times \mathbb{R} \rightarrow \mathcal{B}$ be defined by $\varphi(x, y, p, q, z, \bar{z}, t)=\varphi_{t}(x, y, p, q, z, \bar{z})$. One computes that

$$
\varphi^{*} \theta_{2}=d \bar{z}-(p-\lambda \sin (z+\bar{z})) d x-\left(-q+\lambda^{-1} \sin (z+\bar{z})\right) d y \bmod d t,
$$

where we have set $\lambda=e^{t}$. Thus, by splitting off the $d t$ parts of these forms and relabeling $\varphi^{*} \theta_{2}$ as $\theta_{2}$ on $\mathcal{P}=\mathcal{B} \times \mathbb{R}$, we recover the parametric Bäcklund transformation defined earlier.

Note that it is not clear how to choose the lift $\hat{v}$ so as to make this construction work in general, nor is this question addressed in [10]. However, some light may be shed in 4 , where we will see how this trick applies in another example.

Conservation laws. The space of conservation laws for an EDS $\mathcal{I}$ whose integral manifolds have dimension 2 may be naturally identified with the set of closed 2forms in $\mathcal{I}$, modulo the exterior derivatives of 1 -forms contained in $\mathcal{I}$, i.e., with the quotient space

$$
\frac{\left\{\Phi \in \mathcal{I}^{2} \mid d \Phi=0\right\}}{\left\{d \phi \mid \phi \in \mathcal{I}^{1}\right\}}
$$

Any conservation law for the Monge-Ampère system $\mathcal{I}_{i}=\left\{\theta_{i}, \Theta_{i}, \Psi_{i}\right\}$ has a unique representative of the form

$$
\Phi=Q \Psi_{i}+\theta_{i} \wedge \gamma
$$

for some function $Q$ and 1-form $\gamma$, while any conservation law for the system $\mathcal{J}=$ $\left\{\theta_{1}, \theta_{2}, \Theta_{1}, \Theta_{2}\right\}$ on each slice $\mathcal{B}_{\lambda}$ has a unique representative of the form

$$
\Phi=\theta_{1} \wedge \gamma_{1}+\theta_{2} \wedge \gamma_{2}
$$

for some 1 -forms $\gamma_{1}, \gamma_{2}$ on $\mathcal{B}_{\lambda}$. (It is not difficult to show that any conservation law for the system $\mathcal{J}$ on $\mathcal{B}_{\lambda}$ has a unique lift to a conservation law for the system $\mathcal{K}=\left\{\theta_{1}, \theta_{2}, \zeta\right\}$ on $\mathcal{P}$, and that conversely, any conservation law for $\mathcal{K}$ restricts to a conservation law for $\mathcal{J}$ on each $\mathcal{B}_{\lambda}$. Thus, for ease of computation we will work on $\mathcal{B}$ rather than on $\mathcal{P}$ when computing conservation laws.) Since $\pi_{i}^{*} \mathcal{I}_{i} \subset \mathcal{J}$, the pullback of any conservation law for either of the Monge-Ampère systems $\mathcal{I}_{i}$ to $\mathcal{B}$ is also a conservation law for $\mathcal{J}$, though it typically must be modified by the exterior derivative of a 1-form in $\mathcal{J}$ in order to appear in the form (20).

The condition that $\Phi$ be a closed form in the ideal typically leads to an overdetermined system of PDEs which must be satisfied by the coefficients of $\Phi$. Here we will sketch the computation of the spaces of conservation laws in the sine-Gordon example.

First we compute the conservation laws for the ideal $\mathcal{I}_{1}$. (This will, of course, be isomorphic to the space of conservation laws for $\mathcal{I}_{2}$.) Suppose that

$$
\Phi=Q\left[\left(d p-\frac{1}{2}(\sin z) d y\right) \wedge d x-\left(d q-\frac{1}{2}(\sin z) d x\right) \wedge d y\right]+\theta_{1} \wedge \gamma
$$


is a closed form in $\mathcal{I}_{1}$. Computing $d \Phi \equiv 0 \bmod \theta_{1}$ shows that

$\gamma=-Q_{p} d p+Q_{q} d q-\left(Q_{x}+p Q_{z}+\sin (2 z) Q_{q}\right) d x+\left(Q_{y}+q Q_{z}+\sin (2 z) Q_{p}\right) d y$.

Then the condition $d \Phi=0$ gives a system of second-order PDEs for the function $Q$ whose solutions are

$$
Q=c_{1}(p x-q y)+c_{2} p+c_{3} q
$$

for arbitrary constants $c_{1}, c_{2}, c_{3}$. Thus the space of conservation laws for $\mathcal{I}_{1}$ (and hence for $\mathcal{I}_{2}$ as well) is three-dimensional.

Remark. By applying the method of equivalence to hyperbolic Monge-Ampère systems, one can determine which systems are variational, i.e., are contact-equivalent to the Euler-Lagrange equation for a first-order Lagrangian $\int L(x, y, u, p, q) d x d y$. For those which are variational, Noether's theorem gives a one-to-one correspondence between conservation laws and symmetries for the Monge-Ampère system. The sine-Gordon equation is variational and has a three-dimensional symmetry group, so it is no surprise that we arrive at a three-dimensional space of conservation laws.

Computing the space of conservation laws for $\mathcal{I}$ is considerably more involved. We will use the coframing described above (with $d \lambda=0$ because we are restricting to $\mathcal{B}_{\lambda}$ ), i.e.,

$$
\begin{aligned}
\theta_{1} & =d z-p d x-q d y \\
\theta_{2} & =d \bar{z}-\bar{p} d x-\bar{q} d y \\
\omega^{1} & =d x \\
\omega^{2} & =d \bar{p}-\frac{1}{2} \sin (2 \bar{z}) d y+\lambda \cos (z+\bar{z}) \theta_{2}, \\
\omega^{3} & =d y \\
\omega^{4} & =d q-\frac{1}{2} \sin (2 z) d x-\lambda^{-1} \cos (z-\bar{z}) \theta_{1} .
\end{aligned}
$$

Suppose that

$\Phi=\theta_{1} \wedge\left(P_{1} \omega^{1}+P_{2} \omega^{2}+P_{3} \omega^{3}+P_{4} \omega^{4}\right)+\theta_{2} \wedge\left(Q_{1} \omega^{1}+Q_{2} \omega^{2}+Q_{3} \omega^{3}+Q_{4} \omega^{4}\right)+R \theta_{1} \wedge \theta_{2}$ is a closed form in $\mathcal{J}$. Computing

$$
d \Phi \equiv 0 \quad \bmod \theta_{1}, \theta_{2}
$$

shows that $Q_{1}=P_{1}, Q_{2}=P_{2}, Q_{3}=-P_{3}$, and $Q_{4}=-P_{4}$. Next, computing

$$
d \Phi \equiv 0 \bmod \omega^{1}, \omega^{2}, \theta_{1}-\theta_{2}
$$

shows that

$$
R=\frac{1}{\lambda} \cos (z-\bar{z}) P_{4}-\lambda \cos (z+\bar{z}) P_{2} .
$$

Then the condition $d \Phi=0$ gives a system of 14 first-order PDEs for the four functions $P_{1}, P_{2}, P_{3}, P_{4}$. The compatibility conditions for this system lead to five additional equations; the resulting system is not involutive, so it must be prolonged. (See [11] for details about the Cartan algorithm for linear Pfaffian systems.) Further 
analysis shows that the general solution of this system is

$$
\begin{aligned}
P_{1}= & c_{1}\left[\bar{p}+\frac{1}{2} \lambda \sin (z+\bar{z})-\frac{1}{2} y \sin (2 z)+\lambda(\bar{p} x+q y) \cos (z+\bar{z})\right]+c_{2} \lambda \bar{p} \cos (z+\bar{z}) \\
& \quad+c_{3}\left(\lambda q \cos (z+\bar{z})-\frac{1}{2} \sin (2 z)\right)+\frac{1}{2} c_{4} \lambda \sin (z+\bar{z}), \\
P_{2}= & c_{1} x+c_{2} \\
P_{3}= & c_{1}\left[q-\frac{1}{2} \lambda^{-1} \sin (z-\bar{z})+\frac{1}{2} x \sin (2 \bar{z})+\lambda^{-1}(\bar{p} x-q y) \cos (z-\bar{z})\right] \\
& \quad+c_{2}\left(\lambda^{-1} \bar{p} \cos (z-\bar{z})+\frac{1}{2} \sin (2 \bar{z})\right)-c_{3} \lambda^{-1} q \cos (z-\bar{z})+\frac{1}{2} c_{4} \lambda^{-1} \sin (z-\bar{z}), \\
P_{4}= & c_{1} y+c_{3}
\end{aligned}
$$

for arbitrary constants $c_{1}, c_{2}, c_{3}, c_{4}$. Thus the space of conservation laws for $\mathcal{J}$ is four-dimensional.

Consider the following question: given a conservation law for $\mathcal{I}_{1}$, is its pullback to $\mathcal{J}$ equivalent to the pullback of a conservation law for $\mathcal{I}_{2}$ ? In other words, do the pullbacks of the spaces of conservation laws for $\mathcal{I}_{1}, \mathcal{I}_{2}$ span the same threedimensional subspace of the conservation laws for $\mathcal{J}$ ? Perhaps surprisingly, the answer in this case is no: it can be shown that any conservation law in $\mathcal{I}_{1}$ corresponding to a function $Q$ of the form (21) with $c_{1} \neq 0$ is not equivalent to the pullback of a conservation law for $\mathcal{I}_{2}$, and vice-versa. Thus, the four-dimensional space of conservation laws for $\mathcal{J}$ is the union of the pullbacks of the three-dimensional spaces of conservation laws for $\mathcal{I}_{1}$ and $\mathcal{I}_{2}$.

\section{Goursat's equation}

In an 1899 memoir, Goursat [9] classified those equations of the form $u_{x y}=$ $f\left(x, y, u, u_{x}, u_{y}\right)$ that are integrable, without prolongation, by the method of Darboux (see also [11], Ch. 6). The equation

$$
w_{x y}+2 \frac{\sqrt{w_{x}} \sqrt{w_{y}}}{x+y}=0
$$

occurs at the head of Goursat's list, and we will refer to it here as "Goursat I" or simply as Goursat's equation. (We will restrict our attention to solutions $w(x, y)$ for which the arguments of the square roots in (22) are positive.)

Bäcklund transformation and symmetries. A Bäcklund transformation linking solutions of (22) with those of the wave equation $u_{x y}=0$ was given by Zvyagin [17, and takes the form

$$
u_{x}=\frac{u}{x+y}+\sqrt{2 w_{x}}, \quad u_{y}=\frac{u}{x+y}+\sqrt{2 w_{y}} .
$$

As in the sine-Gordon example, we may use the symmetries of (22) to construct a parametric version of (23). Let $\mathcal{M}_{1}=J^{1}\left(\mathbb{R}^{2}, \mathbb{R}\right)$ with coordinates $x, y, w, p, q$, and $\mathcal{M}_{2}=J^{1}\left(\mathbb{R}^{2}, \mathbb{R}\right)$ with coordinates $\bar{x}, \bar{y}, u, \bar{p}, \bar{q}$. We regard (23), together with $x=\bar{x}$ and $y=\bar{y}$, as defining $\mathcal{B}^{6} \subset \mathcal{M}_{1} \times \mathcal{M}_{2}$. We pull back the contact forms to $\mathcal{B}$ to give the usual rank two Pfaffian system $\mathcal{J}$ generated by

$$
\theta_{1}=d w-p d x-q d y, \quad \theta_{2}=d u-\bar{p} d x-\bar{q} d y,
$$

where

$$
\bar{p}=\frac{u}{x+y}+\sqrt{2 p}, \quad \bar{q}=\frac{u}{x+y}+\sqrt{2 q} .
$$


In an earlier discussion of Darboux integrability ([8], p. 196), Goursat cited (22) as one of the few known Darboux-integrable equations admitting a finitedimensional symmetry group. In fact, its symmetries take the form

$$
w \mapsto A w+B, \quad x \mapsto \frac{a x+b}{c x+d}, \quad y \mapsto-\frac{a y-b}{c y-d}
$$

where $A \neq 0$ and we may take $\left(\begin{array}{ll}a & b \\ c & d\end{array}\right) \in S L(2, \mathbb{R})$. In the case of linear substitution for $w$, or for $x$ and $y$, the vector field on $\mathcal{M}_{1}$ which generates the symmetry has a lift to $\mathcal{B}$ which also covers a symmetry vector field on $\mathcal{M}_{2}$. However, consider the vector field

$$
\mathbf{v}=x^{2} \frac{\partial}{\partial x}-y^{2} \frac{\partial}{\partial y}-2 x p \frac{\partial}{\partial p}+2 y q \frac{\partial}{\partial q},
$$

on $\mathcal{M}_{1}$, which generates the following 1-parameter group of symmetries for (22):

$$
x \mapsto \frac{x}{1-t x}, \quad y \mapsto \frac{y}{1+t y}, \quad p \mapsto(1-t x)^{2} p, \quad q \mapsto(1+t y)^{2} q, \quad w \mapsto w .
$$

Proposition 4.1. There is no lift of $\vee$ to $\mathcal{B}$ which covers a symmetry of the wave equation on $\mathcal{M}_{2}$.

Proof. Suppose

$$
\hat{\mathbf{v}}=x^{2} \frac{\partial}{\partial x}-y^{2} \frac{\partial}{\partial y}-2 x p \frac{\partial}{\partial p}+2 y q \frac{\partial}{\partial q}+f \frac{\partial}{\partial u}
$$

is such a lift, where $f$ is an unknown function of $x, y, w, p, q, u$. The condition $\mathcal{L}_{\hat{v}} \theta_{2} \equiv 0 \bmod \theta_{2}$ implies that

$$
d f=g \theta_{2}+\left(\frac{f}{x+y}+u+x \sqrt{2 p}\right) d x+\left(\frac{f}{x+y}-u-x \sqrt{2 q}\right) d y
$$

where $g$ is necessarily a function of $x, y$ and $u$ only. Further differentiation gives

$$
0=d^{2} f \equiv \frac{x-g}{\sqrt{2 p}} d p \wedge d x-\frac{y+g}{\sqrt{2 q}} d q \wedge d y \quad \bmod d u \wedge d x, d u \wedge d y, d x \wedge d y,
$$

implying that $g=x$ and $g=-y$, a contradiction.

Now let $\varphi_{t}$ be the 1-parameter family of diffeomorphisms of $\mathcal{B}$ defined by 24) along with $u \mapsto u$. By Proposition 4.1 these will not be symmetries of $\mathcal{J}$. Let $\tilde{\mathcal{P}} \subset \mathcal{B} \times \mathbb{R}$ be the open set on which $\varphi_{t}$ is defined, and let $\varphi: \tilde{\mathcal{P}} \rightarrow \mathcal{B}$ be defined by $\varphi(x, y, p, q, u, w, t)=\varphi_{t}(x, y, p, q, u, w)$. Then one computes that

$$
\varphi^{*} \theta_{2} \equiv d u-\left(\frac{u(1+t y)}{x+y}+\sqrt{2 p}\right) \frac{d x}{1-t x}-\left(\frac{u(1-t x)}{x+y}+\sqrt{2 q}\right) \frac{d y}{1+t y} \bmod d t .
$$

This form is the same as the pullback of $\theta_{2}$ to the submanifold $\mathcal{P} \subset \mathcal{M}_{1} \times \mathcal{M}_{2} \times \mathbb{R}$ defined by

$$
(1-t x) \bar{p}=\frac{u(1+t y)}{x+y}+\sqrt{2 p}, \quad(1+t y) \bar{q}=\frac{u(1-t x)}{x+y}+\sqrt{2 q} .
$$

It is straightforward to verify that these equations define a Bäcklund transformation for any $t$ for which the maps (24) are defined. To our knowledge, this parametric transformation is new. 
$G$-structure invariants. The coframe

$$
\begin{array}{rlrl}
\zeta & =((1-t x)(1+t y))^{-1} d t, & \omega^{1}=d x, \\
\theta_{1}=\frac{1}{\sqrt{2 q}}(d w-p d x-q d y), & \omega^{2}=\frac{d p}{\sqrt{2 p}}+\frac{\sqrt{2 q}}{x+y} d y, \\
\theta_{2}=(1-t x)(d u-\bar{p} d x-\bar{q} d y), & \omega^{3}=d y, \\
\omega^{4}=\frac{d q}{\sqrt{2 q}}+\frac{\sqrt{2 p}}{x+y} d x-\left(\frac{u+y \sqrt{2 q}}{1+t y}\right) d t
\end{array}
$$

gives a section of $\mathcal{G}$ with $A_{1}=\sqrt{p / q}$ and $A_{2}=(1-t x) /(1+t y)$. With respect to this coframe, we compute that

$$
\begin{array}{llll}
{\left[\begin{array}{lll}
B_{1} & C_{1} & E_{1} \\
B_{2} & C_{2} & E_{2}
\end{array}\right]=\left[\begin{array}{ccc}
0 & 0 & 0 \\
0 & -(x+y)^{-1} & 0
\end{array}\right],} & {\left[\begin{array}{l}
D_{1} \\
D_{2}
\end{array}\right]=\left[\begin{array}{c}
-(1+t y)(u+x \sqrt{2 p}) \\
0
\end{array}\right],} \\
{\left[\begin{array}{llll}
B_{3} & C_{3} & E_{3} \\
B_{4} & C_{4} & E_{4}
\end{array}\right]=\left[\begin{array}{ccc}
0 & 0 & 0 \\
0 & -(x+y)^{-1} & 1
\end{array}\right],} & {\left[\begin{array}{l}
D_{3} \\
D_{4}
\end{array}\right]=\left[\begin{array}{c}
-(1-t x)(u+y \sqrt{2 q}) \\
0
\end{array}\right] .}
\end{array}
$$

In this case, neither $F_{1}$ nor $F_{2}$ is zero, nor is their ratio a constant. We observe that

(1) Both vectors $\left[\begin{array}{ll}B_{1} & B_{2}\end{array}\right]$ and $\left[\begin{array}{ll}B_{3} & B_{4}\end{array}\right]$ are zero, indicating that, for each value of $t$, this Bäcklund transformation is holonomic (see [6], Thm. 6.2).

(2) Both sets of $B$-, $C$ - and $E$-vectors are linearly dependent, which is consistent with the presence of one-dimensional integrable subsystems in the characteristic systems.

(3) As in the sine-Gordon example, both $D$-vectors are perpendicular to the corresponding $E$-vectors.

Remark. While the transformation (23) is implicit in [17], the transformation given there is actually

$$
z_{x}=\left(\sqrt{w_{x}}+\sqrt{\frac{z-w}{x+y}}\right)^{2}, \quad z_{y}=\left(\sqrt{w_{y}}+\sqrt{\frac{z-w}{x+y}}\right)^{2},
$$

where $z(x, y)$ satisfies the wave equation $z_{x y}=0$ if and only if $w(x, y)$ satisfies (22). Moreover, $z$ is related to $u$ via the change of variable $z=w+\frac{1}{2} u^{2} /(x+y)$, implying that

$$
z_{x}=\frac{1}{2}\left(u_{x}\right)^{2}, \quad z_{y}=\frac{1}{2}\left(u_{y}\right)^{2} .
$$

In other words, the two wave equation solutions $z$ and $u$ are related by the transformation (28). Such transformations can be characterized by the vanishing of all $B$ - and $C$-vectors (see [6], Thm. 3.1).

An interesting observation is that the Bäcklund transformation (27) is clearly the composition of the transformations (23) and (28), each of which are holonomic (e.g., the $B$-vectors are zero). However, one can compute that for (27) the $B$-vectors are nonzero, and therefore (27) is non-holonomic.

Conservation laws. We will compute the spaces of conservation laws for the wave equation, for Goursat's equation (22), and for the ideal $\mathcal{J}$ on $\mathcal{B}_{t}$ for any fixed value of $t$. 
First we compute the conservation laws for the system

$$
\mathcal{I}_{2}=\{d u-\bar{p} d x-\bar{q} d y, d \bar{p} \wedge d x, d \bar{q} \wedge d y\},
$$

which represents the wave equation $u_{x y}=0$. Suppose that

$$
\Phi=Q[d \bar{p} \wedge d x-d \bar{q} \wedge d y]+\theta_{2} \wedge \gamma
$$

is a closed form in $\mathcal{I}_{2}$. Computing $d \Phi \equiv 0 \bmod \theta_{2}$ shows that

$$
\gamma=-Q_{\bar{p}} d \bar{p}+Q_{\bar{q}} d \bar{q}-\left(Q_{x}+\bar{p} Q_{z}\right) d x+\left(Q_{y}+\bar{q} Q_{z}\right) d y .
$$

Then the condition $d \Phi=0$ gives a system of second-order PDEs for the function $Q$ whose solutions are

$$
Q=f(x, \bar{p})+g(y, \bar{q})
$$

for arbitrary functions $f, g$. Thus the space of conservation laws for $\mathcal{I}_{2}$ is infinitedimensional and depends on two arbitrary functions of two variables.

Next we compute the conservation laws for the system

$$
\mathcal{I}_{1}=\left\{d w-p d x-q d y,\left(d p+\frac{2 \sqrt{p q}}{x+y} d y\right) \wedge d x,\left(d q+\frac{2 \sqrt{p q}}{x+y} d x\right) \wedge d y\right\},
$$

which represents Goursat's equation (22). This computation is most easily carried out using the coframe

$$
\begin{aligned}
\theta_{1}=d w-p d x-q d y, & \\
\omega^{1}=d x, & \omega^{3}=d y, \\
\omega^{2}=d p+\frac{2 \sqrt{p q}}{x+y} d y, & \omega^{4}=d q+\frac{2 \sqrt{p q}}{x+y} d x .
\end{aligned}
$$

Suppose that $\Phi=Q\left[\omega^{1} \wedge \omega^{2}-\omega^{3} \wedge \omega^{4}\right]+\theta_{1} \wedge \gamma$ is a closed form in $\mathcal{I}$. Setting

$$
d Q=Q_{0} \theta_{1}+Q_{1} \omega^{1}+Q_{2} \omega^{2}+Q_{3} \omega^{3}+Q_{4} \omega^{4}
$$

and computing $d \Phi \equiv 0 \bmod \theta_{1}$ shows that

$$
\gamma=\left(Q_{1}-\frac{2 \sqrt{p}}{(x+y) \sqrt{q}} Q\right) \omega^{1}+Q_{2} \omega^{2}-\left(Q_{3}-\frac{2 \sqrt{q}}{(x+y) \sqrt{p}} Q\right) \omega^{3}-Q_{4} \omega^{4} .
$$

Then the condition $d \Phi=0$ gives the first-order PDE

$$
Q_{0}=\frac{2 p Q_{2}+2 q Q_{4}+Q}{2(x+y) \sqrt{p q}}
$$

and four additional second-order PDEs for $Q$. The compatibility conditions for this system lead to three additional equations; the resulting system is not involutive, so it must be prolonged. The prolonged system is involutive with the last nonvanishing Cartan character $s_{1}=2$; so by the Cartan-Kähler Theorem (see, e.g., [1]) the space of solutions, and hence the space of conservation laws for (22), depends on two arbitrary functions of one variable. Note that (22) is not variational, so there is no contradiction between the finite-dimensional symmetry group and the infinitedimensional space of conservation laws.

Finally, we compute the space of conservation laws for the system

$$
\mathcal{J}=\left\{\theta_{1}, \theta_{2}, \Theta_{1}, \Theta_{2}\right\}
$$

on $\mathcal{B}_{t}$ for some fixed $t \in \mathbb{R}$. We will use the coframe $\left(\theta_{1}, \theta_{2}, \omega^{1}, \omega^{2}, \omega^{3}, \omega^{4}\right)$ defined by (26) , restricted to $\mathcal{B}$. 
Suppose that

$\Phi=\theta_{1} \wedge\left(P_{1} \omega^{1}+P_{2} \omega^{2}+P_{3} \omega^{3}+P_{4} \omega^{4}\right)+\theta_{2} \wedge\left(Q_{1} \omega^{1}+Q_{2} \omega^{2}+Q_{3} \omega^{3}+Q_{4} \omega^{4}\right)+R \theta_{1} \wedge \theta_{2}$

is a closed form in $\mathcal{J}$. Computing $d \Phi \equiv 0 \bmod \theta_{1}, \theta_{2}$ shows that

$$
\begin{array}{ll}
(1+t y) P_{1}-(1-t x) Q_{1}=0, & \sqrt{p} P_{3}+\sqrt{q} Q_{3}=0, \\
(1+t y) P_{2}-(1-t x) Q_{2}=0, & \sqrt{p} P_{4}+\sqrt{q} Q_{4}=0 .
\end{array}
$$

Thus we may write

$$
\begin{aligned}
\Phi=\left((1-t x) \theta_{1}+(1+t y) \theta_{2}\right) & \wedge\left(S_{1} \omega^{1}+S_{2} \omega^{2}\right) \\
& +\left(\sqrt{p} \theta_{1}-\sqrt{q} \theta_{2}\right) \wedge\left(S_{3} \omega^{3}+S_{4} \omega^{4}\right)+R \theta_{1} \wedge \theta_{2}
\end{aligned}
$$

for some functions $S_{1}, S_{2}, S_{3}, S_{4}$. Computing $d \Phi \equiv 0 \bmod \omega^{1}, \omega^{2}, \sqrt{p} \theta_{1}-\sqrt{q} \theta_{2}$ shows that

$$
R=\frac{(1+t y) S_{2}+\sqrt{q} S_{4}}{x+y} .
$$

Then the condition $d \Phi=0$ gives a system of 14 first-order PDEs for the four functions $S_{1}, S_{2}, S_{3}, S_{4}$. The compatibility conditions for this system lead to three additional equations; the resulting system is not involutive, so it must be prolonged. The prolonged system is involutive with the last nonvanishing Cartan character $s_{2}=2$; so the space of solutions (and hence the space of conservation laws for $\mathcal{J}$ ) depends on two arbitrary functions of two variables.

Clearly, not every conservation law in $\mathcal{J}$ is the pullback of a conservation law for (22), since the space of conservation laws for $\mathcal{J}$ is strictly larger than that for (22). It may be that every conservation law in $\mathcal{J}$ is the pullback of a conservation law for the wave equation, but because the Cartan-Kähler analysis does not give explicit solutions, this hypothesis is difficult to confirm or disprove.

\section{Pseudospherical surfaces in Euclidean space}

In this section we study pseudospherical surfaces as arising from integrals of a Monge-Ampère system. We present the classical Bäcklund transformation in the same context, as a double fibration fitting the description in 9 . We calculate the torsion coefficients for the corresponding $G$-structure, and note how these differ from those in the sine-Gordon case. Since we are also interested in cases where a one-parameter family of Bäcklund transformations may be generated by a symmetry of one of the Monge-Ampère systems involved, we also calculate the symmetries of the system for pseudospherical surfaces.

Surface geometry via moving frames. Let $\mathcal{F}$ be the bundle of Euclidean orthonormal frames on $\mathbb{R}^{3}$, on which the basepoint projection is $\mathbf{x}$ and the vectorvalued functions $e_{1}, e_{2}, e_{3}$ give the members of the frame at $\mathbf{x} \in \mathbb{R}^{3}$. On $\mathcal{F}$ there are canonical 1-forms $\eta^{i}, \eta_{j}^{i}$ defined by resolving the differentials of these vectors in the frame

$$
d \mathbf{x}=e_{i} \eta^{i}, \quad d e_{i}=e_{j} \eta_{i}^{j},
$$

summation being understood on repeated indices. (Because the $e_{i}$ are orthonormal, the $\eta_{j}^{i}$ form a skew-symmetric $3 \times 3$ matrix of 1 -forms.) By differentiating these defining equations we deduce the structure equations for $\mathcal{F}$ :

$$
d \eta^{i}=-\eta_{j}^{i} \wedge \eta^{j}, \quad d \eta_{j}^{i}=-\eta_{k}^{i} \wedge \eta_{j}^{k}
$$


For later use, we note that $\mathcal{F}$ can be identified with a matrix Lie group on which the $\eta$ 's are left-invariant forms. For, we may embed the vectors of the frame in a $4 \times 4$ matrix

$$
g=\left(\begin{array}{ll}
1 & 0 \\
\mathbf{x} & A
\end{array}\right) \in \mathbb{R}^{3} \times S O(3),
$$

where the columns of $A$ are $e_{1}, e_{2}, e_{3}$ in order. Then

$$
g^{-1} d g=\left(\begin{array}{cccc}
0 & 0 & 0 & 0 \\
\eta^{1} & 0 & \eta_{2}^{1} & \eta_{3}^{1} \\
\eta^{2} & \eta_{1}^{2} & 0 & \eta_{3}^{2} \\
\eta^{3} & \eta_{1}^{3} & \eta_{2}^{3} & 0
\end{array}\right) \in \mathbb{R}^{3} \oplus \mathfrak{s} o(3)
$$

The action of this group on $\mathcal{F}$ given by left-multiplication covers the Euclidean motions in $\mathbb{R}^{3}$.

Given a regular surface $S \subset \mathbb{R}^{3}$, it is standard [12] to construct first-order adapted framings along $S$, which are lifts into $\mathcal{F}$,

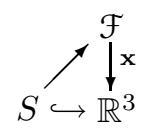

such that $e_{1}, e_{2}$ are tangent and $e_{3}$ is normal to the surface. (Note that such framings are not unique, since they may be modified by rotating $e_{1}, e_{2}$ within the plane they span.) These lifts are surfaces $\Sigma \subset \mathcal{F}$ to which $\eta^{3}$ restricts to be zero, and along which $\eta^{1}, \eta^{2}$ are linearly independent. It follows from the structure equations that

$$
\left.\eta_{i}^{3}\right|_{\Sigma}=h_{i 1} \eta^{1}+h_{i 2} \eta^{2}
$$

for a symmetric $2 \times 2$ matrix of functions $h_{i j}$, which are the components of the second fundamental form of $S$ relative to the basis $e_{1}, e_{2}$. The Gauss curvature $K$ and mean curvature $H$ of the surface are respectively equal to the determinant and one-half the trace of the matrix $\left(h_{i j}\right)$. Consequently, the restricted forms on $\Sigma$ satisfy

$$
\begin{gathered}
\eta_{1}^{3} \wedge \eta_{2}^{3}=K \eta^{1} \wedge \eta^{2} \\
\eta_{1}^{3} \wedge \eta^{2}-\eta_{2}^{3} \wedge \eta^{1}=2 H \eta^{1} \wedge \eta^{2} .
\end{gathered}
$$

The $K=-1$ system. A pseudospherical surface in $\mathbb{R}^{3}$ is one with $K=-1$ at each point. For such surfaces, any first-order adapted framing is an integral surface of the EDS $\mathcal{I}$ generated by the 1 -form $\eta^{3}$ and the 2 -forms $d \eta^{3}$ and

$$
\Psi=\eta_{1}^{3} \wedge \eta_{2}^{3}+\eta^{1} \wedge \eta^{2} .
$$

This would satisfy Definition 1.1 for Monge-Ampère systems, except that the underlying manifold $\mathcal{F}$ is 6 -dimensional. However, the Cartan system of $\mathcal{I}$ is $\left\{\eta^{1}, \eta^{2}, \eta^{3}, \eta_{1}^{3}, \eta_{2}^{3}\right\}$. The integral curves of this Frobenius system are the fibers of a projection $\mathcal{F} \rightarrow \mathcal{U}^{5}$, where $\mathcal{U}$ is the unit tangent bundle of $\mathbb{R}^{3}$ and the projection is given by taking $e_{3}$ as the unit vector. (Consequently, moving along the fibers corresponds to fixing the basepoint of a frame in $\mathcal{F}$ and rotating $e_{1}$ and $e_{2}$.) By a standard argument, the generating differential forms project to become well-defined (up to multiples) on $\mathcal{U}$, and so there is a well-defined EDS on $\mathcal{U}$ such that each integral surface in $\mathcal{F}$ which is transverse to the fibers corresponds to a unique integral 
surface in $\mathcal{U}$. In this case, the EDS on $\mathcal{U}$ is a genuine Monge-Ampère system which we will also call $\mathcal{I}$. That this system is hyperbolic follows from the factorizations

$$
\Psi+d \eta^{3}=\left(\eta_{1}^{3}+\eta^{2}\right) \wedge\left(\eta_{2}^{3}-\eta^{1}\right), \quad \Psi-d \eta^{3}=\left(\eta_{1}^{3}-\eta^{2}\right) \wedge\left(\eta_{2}^{3}+\eta^{1}\right) .
$$

In general, we may perform computations with the generators of $\mathcal{I}$ up on $\mathcal{F}$, knowing that our conclusions will hold on $\mathcal{U}$.

Relationship with sine-Gordon. By breaking the Cauchy characteristic symmetry of the system for pseudospherical surfaces, we can show how such surfaces may be constructed from solutions of the sine-Gordon equation (22).

On an open subset of the surface which is free of umbilic points, we may choose a Darboux framing, i.e., a first-order adapted framing which diagonalizes the second fundamental form. For such a framing, we have

$$
\left(\begin{array}{l}
\eta_{1}^{3} \\
\eta_{2}^{3}
\end{array}\right)=\left(\begin{array}{cc}
\tan \phi & 0 \\
0 & -\cot \phi
\end{array}\right)\left(\begin{array}{l}
\eta^{1} \\
\eta^{2}
\end{array}\right)
$$

where $\phi \in(0, \pi / 2)$ is one-half of the angle between the asymptotic lines. We adjoin $\phi$ as a new variable, and enlarge our EDS to a rank three Pfaffian system $\left\{\eta^{3}, \eta_{1}^{3}-\right.$ $\left.(\tan \phi) \eta^{1}, \eta_{2}^{3}+(\cot \phi) \eta^{2}\right\}$ on $\mathcal{F} \times \mathbb{R}$. The vanishing of the exterior derivatives of the last two 1-forms implies that the forms $\eta^{1} / \cos \phi$ and $\eta^{2} / \sin \phi$ are closed. Hence, on any integral surface there exist local coordinates $t_{1}, t_{2}$ such that $\eta^{1}=(\cos \phi) d t_{1}$ and $\eta^{2}=(\sin \phi) d t_{2}$. It follows that $x=\left(t_{1}+t_{2}\right) / 2$ and $y=\left(t_{1}-t_{2}\right) / 2$ are arclength coordinates along the asymptotic lines.

Substituting $d \phi=\phi_{x} d x+\phi_{y} d y$ into the 2-forms of the EDS shows that $\eta_{2}^{1}=$ $\phi_{y} d y-\phi_{x} d x$ along any integral surface. It is then easy to see that the structure equation $d \eta_{2}^{1}=\eta_{1}^{3} \wedge \eta_{2}^{3}$ implies that $\phi$ satisfies the sine-Gordon equation

$$
\phi_{x y}=\frac{1}{2} \sin (2 \phi) \text {. }
$$

Conversely, if $\phi(x, y)$ is a solution to the sine-Gordon equation, we may produce a pseudospherical surface by integration. For, if we let $A=\left(e_{1}, e_{2}, e_{3}\right)$ as before, then the structure equations $d e_{i}=e_{j} \eta_{i}^{j}$ imply that

$$
\frac{\partial A}{\partial x}=A\left(\begin{array}{ccc}
0 & -\phi_{x} & -\sin \phi \\
\phi_{x} & 0 & \cos \phi \\
\sin \phi & -\cos \phi & 0
\end{array}\right), \quad \frac{\partial A}{\partial y}=A\left(\begin{array}{ccc}
0 & \phi_{y} & -\sin \phi \\
-\phi_{y} & 0 & -\cos \phi \\
\sin \phi & \cos \phi & 0
\end{array}\right) .
$$

(This is an overdetermined system for matrix $A(x, t)$, and its integrability condition is the sine-Gordon equation for $\phi$.) So, given a solution to sine-Gordon, we may obtain the framing by solving linear systems of ODE, and then solve for the surface $\mathbf{x}(x, y) \in \mathbb{R}^{3}$ by integrating the equations

$$
\frac{\partial \mathbf{x}}{\partial x}=e_{1} \cos \phi+e_{2} \sin \phi, \quad \frac{\partial \mathbf{x}}{\partial y}=e_{1} \cos \phi-e_{2} \sin \phi .
$$

For example, the 1-soliton solution $u=4 \arctan \left(\exp \left(a x+a^{-1} y\right)\right), a \neq 0$, gives the classical pseudosphere (i.e., the surface generated by revolving the tractrix about its asymptote) when $a^{2}=1$ and gives Dini's surface when $a^{2} \neq 1$.

Remark. The relationship between solutions of the Monge-Ampère system for pseudospherical surfaces and solutions of sine-Gordon can be described in terms of a double fibration that is similar to the geometric definition for Bäcklund transformations given in $₫ \mathbb{1}$ For, let $\mathcal{N}=J^{1}\left(\mathbb{R}^{2}, \mathbb{R}\right)$ carry the sine-Gordon system given in 
93, and let $\mathcal{U}$ be the 5 -dimensional quotient manifold on which $\mathcal{I}$ lives. Then there is a double fibration

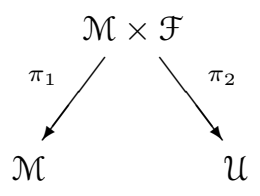

with the obvious projections. Moreover, (31) and (32) are equivalent to a Pfaffian system $\mathcal{S}$ of rank six on $\mathcal{M} \times \mathcal{F}$, with the property that if $\Sigma \subset \mathcal{M}$ is any integral surface of the sine-Gordon system, then $\mathcal{S}$ restricts to be Frobenius on $\mathcal{N}_{\Sigma}=\pi_{1}^{-1}(\Sigma)$. Thus, $\mathcal{N}_{\Sigma}$ is foliated by integral surfaces of $\mathcal{S}$, each of which projects via $\pi_{2}$ to give an integral surface of $\mathcal{I}$. For this reason, we say that the pseudospherical system is an integrable extension (see [2] or Ch. 6 of [11]) of the sine-Gordon system. However, the double fibration described here does not give a Bäcklund transformation, in the sense of $\$ 1$ between the two Monge-Ampère systems.

Bäcklund transformation. The Bäcklund transformation for pseudospherical surfaces arises naturally in a classical context, the study of line congruences in Euclidean space. A line congruence is a two-parameter family of lines; associated to the congruence are two focal surfaces to which each line in the family is tangent. Leaving degeneracies aside, locally the lines give a 1-to-1 correspondence between points on the focal surfaces.

Theorem (Bäcklund). If the distance $\lambda$ between corresponding points on the focal surfaces and the angle $\psi$ between the surface normal at corresponding points are both constant, then the two surfaces have the same constant negative Gauss curvature, and we say they are related by a Bäcklund transformation.

(For a proof using moving frames, see [4].)

For the rest of this section, we normalize the Gauss curvature to be -1 . Then $\lambda=$ $\sin \psi$, and we may regard $\lambda$ or $\psi$ as the parameter in the Bäcklund transformation.

Let $\mathcal{F}$ and $\overline{\mathcal{F}}$ be two copies of the frame bundle. The starting point of the proof of Bäcklund's Theorem is adapting frames $\left(\mathbf{x}, e_{1}, e_{2}, e_{3}\right)$ and $\left(\overline{\mathbf{x}}, \bar{e}_{1}, \bar{e}_{2}, \bar{e}_{3}\right)$ along the two surfaces so that $e_{1}=\bar{e}_{1}$ is tangent to the line connecting corresponding points $\mathbf{x}$ and $\overline{\mathbf{x}}$. Then the graph of the Bäcklund transformation is a 6-dimensional submanifold of $\mathcal{F} \times \overline{\mathcal{F}}$ on which

$$
\begin{aligned}
\overline{\mathbf{x}} & =\mathbf{x}+\lambda e_{1}, \\
\bar{e}_{1} & =e_{1}, \\
\bar{e}_{2} & =e_{2} \cos \psi+e_{3} \sin \psi, \\
\bar{e}_{3} & =e_{3} \cos \psi-e_{2} \sin \psi
\end{aligned}
$$

for some constant $\lambda=\sin \psi$.

We will regard (33) as defining a 7-dimensional submanifold $\mathcal{P} \subset \mathcal{F} \times \overline{\mathcal{F}} \times \mathbb{R}$. Differentiating the above equations reveals the following relationships between the 
canonical forms:

$$
\begin{aligned}
& \bar{\eta}^{1}=\eta^{1}+d \lambda, \quad \bar{\eta}_{3}^{2}=\eta_{3}^{2}-d \psi, \\
& \left(\begin{array}{l}
\bar{\eta}^{2} \\
\bar{\eta}^{3}
\end{array}\right)=\left(\begin{array}{cc}
\cos \psi & \sin \psi \\
-\sin \psi & \cos \psi
\end{array}\right)\left(\begin{array}{l}
\eta^{2}-\lambda \eta_{2}^{1} \\
\eta^{3}-\lambda \eta_{3}^{1}
\end{array}\right) \text {, } \\
& \left(\begin{array}{c}
\bar{\eta}_{2}^{1} \\
\bar{\eta}_{3}^{1}
\end{array}\right)=\left(\begin{array}{cc}
\cos \psi & \sin \psi \\
-\sin \psi & \cos \psi
\end{array}\right)\left(\begin{array}{l}
\eta_{2}^{1} \\
\eta_{3}^{1}
\end{array}\right) .
\end{aligned}
$$

$G$-structure invariants. On $\mathcal{P}$ we will choose $\theta_{1}, \theta_{2}$ to be multiples of $\eta^{3}, \bar{\eta}^{3}$ respectively, and let $\mathcal{K}=\left\{\eta^{3}, \bar{\eta}^{3}, d \psi\right\}$. That (33) really does define a Bäcklund transformation for pseudospherical surfaces follows from

$$
d \bar{\eta}^{3} \equiv \eta_{1}^{3} \wedge \eta_{2}^{3}+\eta^{1} \wedge \eta^{2} \bmod \mathcal{K}
$$

Moreover, this shows us how we may choose a section of the $G$-structure $\mathcal{G}$ on $\mathcal{P}$ associated to the Bäcklund transformation. The coframe

$$
\begin{array}{rlrl}
\zeta & =d \psi, & \omega^{1}=\eta^{1}-\eta_{3}^{2}, \\
\theta_{1}=2 \eta^{3}, & \omega^{2}=\eta^{2}+\eta_{3}^{1}, \\
\theta_{2}=2 \bar{\eta}^{3}, & \omega^{3}=\eta^{1}+\eta_{3}^{2}, \\
\omega^{4}=\eta^{2}-\eta_{3}^{1}
\end{array}
$$

satisfies (18) with $A_{1}=-1$ and $A_{2}=1$. If we modify this coframe by setting

$$
\begin{aligned}
& \omega^{2}=\eta^{2}+\eta_{3}^{1}-\frac{1+\cos \psi}{\sin \psi} \eta^{3}, \\
& \omega^{3}=\bar{\eta}^{1}+\bar{\eta}_{3}^{2}, \\
& \omega^{4}=\eta^{2}-\eta_{3}^{1}+\frac{1-\cos \psi}{\sin \psi} \eta^{3}=\bar{\eta}^{2}-\bar{\eta}_{3}^{1}-\frac{1-\cos \psi}{\sin \psi} \bar{\eta}^{3},
\end{aligned}
$$

then we get a section of $\mathcal{G}$. Using this modified coframe, we compute

$$
\begin{array}{lll}
{\left[\begin{array}{lll}
B_{1} & C_{1} & E_{1} \\
B_{2} & C_{2} & E_{2}
\end{array}\right]=\frac{1}{4(1-\cos \psi)}\left[\begin{array}{ccc}
\csc \psi & 0 & 0 \\
0 & -2 \sin \psi & 2
\end{array}\right],} & {\left[\begin{array}{l}
D_{1} \\
D_{2}
\end{array}\right]=\left[\begin{array}{c}
0 \\
1+\cos \psi
\end{array}\right],} \\
{\left[\begin{array}{lll}
B_{3} & C_{3} & E_{3} \\
B_{4} & C_{4} & E_{4}
\end{array}\right]=\frac{-1}{4(1+\cos \psi)}\left[\begin{array}{ccc}
\csc \psi & 0 & 0 \\
0 & 2 \sin \psi & 2
\end{array}\right],} & {\left[\begin{array}{l}
D_{3} \\
D_{4}
\end{array}\right]=\left[\begin{array}{c}
0 \\
1-\cos \psi
\end{array}\right] .}
\end{array}
$$

Again, $F_{1}$ and $F_{2}$ are nonzero, and their ratio is non-constant. We observe that

(1) The vector pairs $\left\{\left[B_{1} B_{2}\right],\left[C_{1} C_{2}\right]\right\}$ and $\left\{\left[B_{3} B_{4}\right],\left[C_{3} C_{4}\right]\right\}$ are both linearly independent, by contrast with the sine-Gordon example.

(2) As in the sine-Gordon example, the vectors $\left[\begin{array}{ll}D_{1} & D_{2}\end{array}\right]$ and $\left[\begin{array}{ll}D_{3} & D_{4}\end{array}\right]$ are perpendicular to their respective $B$-vectors, and the $E$-vectors are dependent on their respective $C$-vectors.

Symmetries. For the pseudospherical surface system on $\mathcal{F}$, the Cauchy characteristic vector fields correspond to infinitesimal rotation of the frame within the $e_{1} e_{2}$ plane. Other symmetries generate the six-dimensional group of Euclidean motions of $\mathbb{R}^{3}$, and these vector fields are precisely the right-invariant vector fields on $\mathcal{F}$ as a Lie group.

Proposition 5.1. These are the only symmetries of the pseudospherical system $\mathcal{I}$. 
Proof. Choose a finite-dimensional complement to $\mathfrak{C}_{\mathcal{I}} \subset \mathfrak{S}_{\mathcal{I}}$ by requiring that the symmetry vector field satisfy $\vee\lrcorner \eta_{2}^{1}=0$. We will show that the space of such symmetries is 6-dimensional, and hence consists only of the projections of the rightinvariant vector fields into the complementary subspace.

Suppose that

$$
\left.\vee\lrcorner \eta^{3}=x, \quad \vee\right\lrcorner \eta^{i}=a_{i}, \quad \vee \bullet \eta_{i}^{3}=b_{i}
$$

for $i=1,2$. Then the requirement that $\mathcal{L}_{\mathrm{v}} \eta^{3} \in \mathcal{I}$ implies that

$$
d x \equiv a_{i} \eta_{3}^{i}+b_{i} \eta^{i} \bmod \eta^{3} .
$$

Therefore, let $c$ be the $\eta^{3}$-component of $d x$. Differentiation gives

$$
0=d(d x)=\alpha_{i} \wedge \eta_{3}^{i}+\beta_{i} \wedge \eta^{i}+\gamma \wedge \eta^{3}
$$

where $\alpha_{i}=d a_{i}-a_{j} \eta_{i}^{j}, \beta_{i}=d b_{i}-b_{j} \eta_{i}^{j}-c \eta_{i}^{3}, \gamma=d c-b_{i} \eta_{3}^{i}$, and all indices range between 1 and 2. By the Cartan Lemma [1], there must be a symmetric matrix $S$ of functions such that

$$
{ }^{t}\left(\alpha_{1}, \alpha_{2}, \beta_{1}, \beta_{2}, \gamma\right)=S^{t}\left(\eta_{3}^{1}, \eta_{3}^{2}, \eta^{1}, \eta^{2}, \eta^{3}\right) .
$$

Let the entries of $S$ be $S_{\mu \nu}=S_{\nu \mu}$ with indices between 1 and 5 . The additional symmetry condition is $\mathcal{L}_{\mathrm{v}} \Psi \equiv 0 \bmod \mathcal{I}$. One computes that

$$
\begin{aligned}
\mathcal{L}_{\vee} \Psi \equiv & 2\left(S_{13}+S_{24}-c\right) \eta^{1} \wedge \eta^{2}+2\left(S_{12}+S_{34}\right) \eta_{3}^{1} \wedge \eta^{1} \\
& +\left(S_{11}-S_{44}+x\right) \eta_{3}^{1} \wedge \eta^{2}-\left(S_{22}-S_{33}+x\right) \eta_{3}^{2} \wedge \eta^{1}
\end{aligned}
$$

modulo $\eta^{3}, d \eta^{3}, \Psi$. Therefore, we must have $S_{24}=c-S_{13}, S_{34}=-S_{12}, S_{44}=$ $S_{11}+x$ and $S_{33}=S_{22}+x$. We may take (34) and (35) as defining a Pfaffian system of rank 6 . Upon calculating the prolongation of this system, we find that the only integral manifolds satisfying the independence condition $\eta_{3}^{1} \wedge \eta_{3}^{2} \wedge \eta^{1} \wedge \eta^{2} \wedge \eta^{3} \neq 0$ have $c=0$, and satisfy the rank 6 Frobenius system defined by (34) and

$$
\begin{aligned}
d a_{1} & =-x \eta_{3}^{1}+S_{14} \eta^{2}-b_{1} \eta^{3}-a_{2} \eta_{2}^{1}, \\
d a_{2} & =-x \eta_{3}^{2}-S_{14} \eta^{1}-b_{2} \eta^{3}+a_{1} \eta_{2}^{1}, \\
d b_{1} & =-S_{14} \eta_{3}^{2}-b_{2} \eta_{2}^{1}, \\
d b_{2} & =S_{14} \eta_{3}^{1}+b_{1} \eta_{2}^{1}, \\
d S_{14} & =b_{1} \eta_{3}^{2}-b_{2} \eta_{3}^{1} .
\end{aligned}
$$

Since any Frobenius system of rank $k$ is locally equivalent to a system of $k$ firstorder ODE, it follows that the space of such symmetries is parametrized by six constants, and hence is spanned by the projections of the Euclidean symmetries into the complement of $\mathfrak{C}_{\mathcal{I}}$.

Conservation laws. First we compute the space of conservation laws for the ideal $\mathcal{I}_{1}$. (This will, of course, be isomorphic to the space of conservation laws for $\mathcal{I}_{2}$.) Suppose that

$$
\Phi=Q\left[\eta_{1}^{3} \wedge \eta_{2}^{3}+\eta^{1} \wedge \eta^{2}\right]+\eta^{3} \wedge \gamma
$$

is a closed form in $\mathcal{I}_{1}$. Setting

$$
d Q=Q_{1} \eta^{1}+Q_{2} \eta^{2}+Q_{3} \eta^{3}+Q_{4} \eta_{1}^{3}+Q_{5} \eta_{2}^{3}
$$

and computing $d \Phi \equiv 0 \bmod \eta^{3}$ shows that

$$
\gamma=-Q_{5} \eta^{1}+Q_{4} \eta^{2}+Q_{2} \eta_{1}^{3}-Q_{1} \eta_{2}^{3} .
$$


Then the condition $d \Phi=0$ gives the first-order PDE

$$
Q_{3}=0
$$

and four additional second-order PDEs for $Q$. The compatibility conditions for this system lead to five additional equations; the resulting system is not involutive, so it must be prolonged. The resulting system is a Frobenius Pfaffian system of rank six; thus the space of solutions (and hence the space of conservation laws for each of $\mathcal{I}_{1}$ and $\left.\mathcal{I}_{2}\right)$ is 6-dimensional. This is the expected result: the pseudospherical system is variational, so by Noether's theorem there is a one-to-one correspondence between conservation laws and symmetries.

Next we compute the space of conservation laws for $\mathcal{J}$, using the coframing described above, i.e.,

$$
\begin{aligned}
\theta_{1} & =2 \eta^{3}, & \theta_{2} & =2 \bar{\eta}^{3}, \\
\omega^{1} & =\eta^{1}-\eta_{3}^{2}, & \omega^{3} & =\bar{\eta}^{1}+\bar{\eta}_{3}^{2}, \\
\omega^{2} & =\eta^{2}+\eta_{3}^{1}-\frac{1+\cos \psi}{\sin \psi} \eta^{3}, & \omega^{4} & =\eta^{2}-\eta_{3}^{1}+\frac{1-\cos \psi}{\sin \psi} \eta^{3} .
\end{aligned}
$$

Suppose that

$\Phi=\theta_{1} \wedge\left(P_{1} \omega^{1}+P_{2} \omega^{2}+P_{3} \omega^{3}+P_{4} \omega^{4}\right)+\theta_{2} \wedge\left(Q_{1} \omega^{1}+Q_{2} \omega^{2}+Q_{3} \omega^{3}+Q_{4} \omega^{4}\right)+R \theta_{1} \wedge \theta_{2}$

is a closed form in $\mathcal{J}$. Computing $d \Phi \equiv 0 \bmod \theta_{1}, \theta_{2}$ shows that $Q_{1}=-P_{1}$, $Q_{2}=-P_{2}, Q_{3}=P_{3}$, and $Q_{4}=P_{4}$. Next, computing $d \Phi \equiv 0 \bmod \omega^{3}, \omega^{4}, \theta_{1}-\theta_{2}$ shows that

$$
R=\frac{(1+\cos \psi 1) P_{2}+(1-\cos \psi) P_{4}}{2 \sin \psi} .
$$

Then the condition $d \Phi=0$ gives a system of 14 first-order PDEs for the four functions $P_{1}, P_{2}, P_{3}, P_{4}$. The compatibility conditions for this system lead to seven additional equations; the resulting system is not involutive, so it must be prolonged. The resulting system is a Frobenius Pfaffian system of rank seven; thus the space of solutions (and hence the space of conservation laws for $\mathcal{I}_{1}$ ) is 7-dimensional.

Now we ask the same question as in the sine-Gordon example: given a conservation law for $\mathcal{I}_{1}$, is its pullback to $\mathcal{J}$ equivalent to the pullback of a conservation law for $\mathcal{I}_{2}$ ? By contrast with that case, the answer here is yes. We can explain this intuitively using the duality between conservation laws and symmetries for variational Monge-Ampère systems. The six independent symmetries of $\mathcal{I}_{1}$ are all symmetries of $\mathcal{B}$ as well, unlike in the sine-Gordon case where the Lie transformation $L_{\mu}$ is not a symmetry of $\mathcal{B}$. Thus the pullbacks of the spaces of conservation laws for $\mathcal{I}_{1}$ and $\mathcal{I}_{2}$ span the same 6-dimensional subspace of the 7-dimensional space of conservation laws for $\mathcal{J}$. The significance of the "extra" conservation law for $\mathcal{J}$ is not clear; we hope to explore this issue in the future.

\section{Timelike CMC surfaces in Lorentzian QuAdrics}

In this section we study parametric Bäcklund transformations for timelike surfaces of constant mean curvature in the standard negatively curved Lorentzian space form. This example will show several similarities to the pseudospherical example, but exhibits values for the Bäcklund invariants which are different from any of the previous examples. Before computing these invariants, we will briefly review surface theory in this setting and construct the Bäcklund transformation. 
Let $\mathbf{L}^{2,2}$ denote $\mathbb{R}^{4}$ equipped with an inner product with ++-- signature, and let $\mathbf{H}^{2,1}$ be the quadric hypersurface in $\mathbf{L}^{2,2}$ defined by $\langle\mathbf{x}, \mathbf{x}\rangle=-1$. Then $\mathbf{H}^{2,1}$ inherits a Lorentzian metric from $\mathbf{L}^{2,2}$ and is a homogeneous space form under the action of $O(2,2)$. Let $\mathcal{F}$ be the bundle of orthogonal frames $\left(\mathbf{x}, e_{1}, e_{2}, e_{3}\right)$ such that $\mathbf{x} \in \mathbf{H}^{2,1}$ and $e_{i} \in T_{\mathbf{x}} \mathbf{H}^{2,1}$, with $\left\langle e_{1}, e_{1}\right\rangle=\left\langle e_{3}, e_{3}\right\rangle=1$ and $\left\langle e_{2}, e_{2}\right\rangle=-1$. On $\mathcal{F}$ we define canonical 1-forms $\eta^{i}, \eta_{j}^{i}$ satisfying

$$
d \mathbf{x}=e_{i} \eta^{i}, \quad d e_{i}=\mathbf{x} \eta_{j}^{0}+e_{j} \eta_{i}^{j},
$$

where $\eta_{1}^{0}=\eta^{1}, \eta_{2}^{0}=-\eta^{2}, \eta_{3}^{0}=\eta^{3}, \eta_{2}^{1}=\eta_{1}^{2}, \eta_{3}^{1}=-\eta_{1}^{3}, \eta_{3}^{2}=\eta_{2}^{3}$, and the diagonal $\eta_{j}^{i}$ 's are zero. The structure equations

$$
d \eta^{i}=-\eta_{j}^{i} \wedge \eta^{j}, \quad d \eta_{j}^{i}=-\eta_{k}^{i} \wedge \eta_{j}^{k}-\eta^{i} \wedge \eta_{j}^{0}
$$

imply that all planes in $\mathbf{H}^{2,1}$ have sectional curvature -1 (see [13] for curvature conventions).

A timelike surface $S \subset \mathbf{H}^{2,1}$ is one to which the metric restricts to have +signature. A first-order adapted framing on $S$ is a (local) lift into $\mathcal{F}$ such that $e_{3}$ is normal to the tangent plane of $S$. Such lifts may be modified by a Lorentzian rotation of $e_{1}$ and $e_{2}$. Along such a lift $\Sigma \subset \mathcal{F}$ we have $\eta^{3}=0$, and hence (30) holds for a symmetric matrix of functions $h_{i j}$. Computing the change in $h_{i j}$ under rotations shows that $\operatorname{det}\left(h_{i j}\right)$ and $h_{11}-h_{22}$ are invariants. In fact, the Gauss and mean curvature of $S$ are defined by

$$
\begin{aligned}
d \eta_{2}^{1}=-K \eta^{1} \wedge \eta^{2} & =-\eta_{3}^{1} \wedge \eta_{2}^{3}+\eta^{1} \wedge \eta^{2}, \\
2 H \eta^{1} \wedge \eta^{2} & =\eta_{1}^{3} \wedge \eta^{2}+\eta_{2}^{3} \wedge \eta^{1} .
\end{aligned}
$$

The CMC system. Suppose $S$ has constant mean curvature $H$. Then $\Sigma$ is an integral surface of the Monge-Ampère system generated by $\eta^{3}, d \eta^{3}$ and

$$
\Psi=\eta_{3}^{1} \wedge \eta^{2}-\eta_{3}^{2} \wedge \eta^{1}+2 H \eta^{1} \wedge \eta^{2} .
$$

Integral surfaces of this system correspond to solutions of a certain second-order hyperbolic PDE in local coordinates on $S$ obtained by choosing first-order adapted frames which diagonalize $h_{i j}$. For, suppose that $h_{12}=0, h_{11}=e^{2 u}+H$ and $h_{22}=e^{2 u}-H$ for some function $u$. Differentiating (30) shows that $e^{u} \eta^{1}$ and $e^{u} \eta^{2}$ are closed, and hence equal to $d x$ and $d y$, respectively, for some functions $x$ and $y$. Determining $\eta_{2}^{1}$ in terms of $u$ and substituting in the Gauss equation (36) shows that, as a function of the local coordinates $x$ and $y, u$ satisfies the equation

$$
u_{x x}-u_{y y}=e^{2 u}+\left(1-H^{2}\right) e^{-2 u} .
$$

This is equivalent to the sinh-Gordon equation when $H^{2}>1$, the cosh-Gordon equation when $H^{2}<1$, and to Liouville's equation when $H^{2}=1$. Given a solution $u(x, y)$ of one of these equations defined on an open set $U \subset \mathbb{R}^{2}$, we can construct the corresponding CMC immersion by integrating a compatible system of total differential equations such as (31) and (321). (In practice, the immersion will only be defined on a sufficiently small neighbourhood of a given point in $U$.)

Remark. The correspondence between linear Weingarten surfaces in Riemannian and semi-Riemannian space forms and solutions of a handful of "model" secondorder PDEs is set out in detail in the dissertation of Penn [14]. 
Bäcklund transformation. A geometrically-defined Bäcklund transformation for timelike CMC surfaces is provided by the following analogue of Bäcklund's theorem, which is also a generalization of a Bäcklund theorem for timelike minimal surfaces in flat Minkowski space [5].

Theorem 1. Let $U \subset \mathbb{R}^{2}$ be an open set and suppose $\mathbf{x}, \overline{\mathbf{x}}: U \rightarrow \mathbf{H}^{2,1}$ are two timelike immersions with unit normal vectors $e_{3}$ and $\bar{e}_{3}$ respectively, such that

(i) for each $p \in U, \mathbf{x}(p)$ and $\overline{\mathbf{x}}(p)$ are the endpoints of a spacelike geodesic of fixed length $r>0$

(ii) at each end, the tangent vectors to the geodesic orthogonally project to null vectors in the tangent spaces to the surfaces $S=\mathbf{x}(U), \bar{S}=\overline{\mathbf{x}}(U)$;

(iii) the inner product of the parallel transport of $e_{3}$ along the geodesic from $\mathbf{x}$ to $\overline{\mathbf{x}}$ with the aforementioned null vector in $T \bar{S}$ is a constant $h \neq 0$.

Then both surfaces have constant mean curvature $H= \pm \frac{2-h}{2 \tanh r}$.

Proof. We may choose first-order adapted frames at corresponding points on the surface so that $e_{1}+e_{2}+e_{3}$ and $\bar{e}_{1}+\bar{e}_{2}+\bar{e}_{3}$ are unit tangents to the geodesic which are related by parallel transport along the geodesic. It follows that the adapted frames must be related by equations of the form

$$
\left(\begin{array}{c}
\overline{\mathbf{x}} \\
\bar{e}_{3} \\
\bar{e}_{1} \\
\bar{e}_{2}
\end{array}\right)=\left(\begin{array}{cccc}
c & s & s & s \\
s & (1-h) c & c & (1-h) c \\
s & c & (1-k) c & (1-k) c \\
-s & (h-1) c & (k-1) c & (h+k-1) c
\end{array}\right)\left(\begin{array}{c}
\mathbf{x} \\
e_{3} \\
e_{1} \\
e_{2}
\end{array}\right)
$$

where $c=\cosh r, s=\sinh r$, and $c^{2} h k=1$. Computing and comparing the canonical forms shows that

$$
0=d \bar{\eta}^{3}=\bar{\eta}_{3}^{1} \wedge \bar{\eta}^{1}-\bar{\eta}_{3}^{2} \wedge \bar{\eta}^{2}=\eta_{3}^{1} \wedge \eta^{2}-\eta_{3}^{2} \wedge \eta^{1}+\frac{2-h}{\tanh r} \eta^{1} \wedge \eta^{2}
$$

Thus, surface $S$ has the desired mean curvature, and the mean curvature of $\bar{S}$ follows by symmetry. (Note, however, that the sign of the mean curvature depends on the orientation of the normal $e_{3}$ relative to $e_{1}$ and $e_{2}$. Since the limit of (37) as $r \rightarrow 0$ interchanges $e_{1}$ and $e_{3}$, the sign of the curvature of the surface $\bar{S}$, using normal $\bar{e}_{3}$, is opposite from that of $S$.)

Thus, for any fixed value of $H$, there is a one-parameter family of Bäcklund transformations for timelike CMC surfaces in $\mathbf{H}^{2,1}$. (Note that we may also allow $r<0$ as well as $r>0$ in (37).) For the rest of this section, we will restrict to $H=1$, and so we will set

$$
h=2(1+\tanh r), \quad k=\frac{1}{2}(1-\tanh r) .
$$


As in \$5] we regard (37) as defining a 7-dimensional submanifold $\mathcal{P} \subset \mathcal{F} \times \overline{\mathcal{F}} \times \mathbb{R}$. Differentiation shows that the canonical forms, restricted to $\mathcal{P}$, satisfy

$$
\begin{aligned}
\left(\begin{array}{l}
\bar{\eta}^{1}-\bar{\eta}^{2} \\
\bar{\eta}_{3}^{2}-\bar{\eta}_{3}^{1}
\end{array}\right) & =\frac{1}{\lambda}\left(\begin{array}{cc}
1+\lambda & 1-\lambda \\
1-\lambda & 1+\lambda
\end{array}\right)\left(\begin{array}{l}
\eta^{3}-\eta^{2} \\
\eta_{2}^{1}+\eta_{3}^{1}
\end{array}\right), \quad \lambda=e^{2 r} \neq 1, \\
\left(\begin{array}{ll}
\bar{\eta}^{3}-\bar{\eta}^{2} \\
\bar{\eta}_{2}^{1}+\bar{\eta}_{3}^{1}
\end{array}\right) & =\frac{1}{4}\left(\begin{array}{ll}
1+\lambda & 1-\lambda \\
1-\lambda & 1+\lambda
\end{array}\right)\left(\begin{array}{l}
\eta^{1}-\eta^{2} \\
\eta_{3}^{2}-\eta_{3}^{1}
\end{array}\right), \\
\bar{\eta}^{1}+\bar{\eta}^{3}-\bar{\eta}^{2} & =\eta^{1}+\eta^{3}-\eta^{2}+d r, \\
\bar{\eta}_{2}^{1}+\bar{\eta}_{3}^{1}-\bar{\eta}_{3}^{2} & =-\left(\eta_{2}^{1}+\eta_{3}^{1}-\eta_{3}^{2}+d r\right) .
\end{aligned}
$$

$G$-structure invariants. On $\mathcal{P}$, let $\mathcal{K}=\left\{\eta^{3}, \bar{\eta}^{3}, d r\right\}$. The above relations imply that

$$
d \bar{\eta}^{3} \equiv\left(\eta_{3}^{1}+\eta^{1}\right) \wedge \eta^{2}-\left(\eta_{3}^{2}+\eta^{2}\right) \wedge \eta^{1} \bmod \mathcal{K}
$$

while

$$
d \eta^{3} \equiv\left(\eta_{3}^{1}+\eta^{1}\right) \wedge \eta^{1}-\left(\eta_{3}^{2}+\eta^{2}\right) \wedge \eta^{2} \bmod \mathcal{K}
$$

Thus, the coframe

$$
\begin{array}{rlrl}
\zeta & =d r, & \omega^{1}=\eta^{1}-\eta^{2}, \\
\theta_{1}=2 \eta^{3}, & \omega^{2}=\eta_{3}^{2}+\eta^{2}+\eta_{3}^{1}+\eta^{1}, \\
\theta_{2}=2 \bar{\eta}^{3}, & \omega^{3}=\eta^{1}+\eta^{2}, \\
\omega^{4}=\eta_{3}^{2}+\eta^{2}-\eta_{3}^{1}-\eta^{1}
\end{array}
$$

satisfies (18) with $A_{1}=-1$ and $A_{2}=1$. If we modify this coframe by setting

$$
\begin{aligned}
& \omega^{1}=\eta^{1}-\eta^{2}+\eta^{3}, \\
& \omega^{3}=\eta^{1}+\eta^{2}-\eta^{3}+d r, \\
& \omega^{4}=\eta_{3}^{2}-\eta_{3}^{1}-\eta^{1}+\eta^{2}+\frac{2}{\lambda-1}\left(\eta^{3}+d r\right),
\end{aligned}
$$

then we get a section of $\mathcal{G}$. With respect to this coframe, we compute

$$
\begin{array}{lll}
{\left[\begin{array}{lll}
B_{1} & C_{1} & E_{1} \\
B_{2} & C_{2} & E_{2}
\end{array}\right]=\frac{1}{4(\lambda-1)}\left[\begin{array}{ccc}
\lambda & 2(\lambda-1) & 2 \lambda \\
0 & 0 & 0
\end{array}\right],} & {\left[\begin{array}{l}
D_{1} \\
D_{2}
\end{array}\right]=\left[\begin{array}{l}
0 \\
1
\end{array}\right],} \\
{\left[\begin{array}{lll}
B_{3} & C_{3} & E_{3} \\
B_{4} & C_{4} & E_{4}
\end{array}\right]=\frac{1}{4(\lambda-1)}\left[\begin{array}{ccc}
\lambda & 2(\lambda-1) & -2 \lambda \\
\frac{2 \lambda}{\lambda-1} & -4 & \frac{4 \lambda}{\lambda-1}
\end{array}\right],} & {\left[\begin{array}{c}
D_{3} \\
D_{4}
\end{array}\right]=\left[\begin{array}{c}
\frac{2}{\lambda-1} \\
-1
\end{array}\right] .}
\end{array}
$$

As well, $F_{1}=2 /(\lambda-1)$ and $F_{2}=0$. In this example, we observe that

(1) The vectors $\left\{\left[\begin{array}{ll}B_{1} & B_{2}\end{array}\right],\left[\begin{array}{ll}C_{1} & C_{2}\end{array}\right]\right\}$ are linearly dependent, while $\left\{\left[\begin{array}{ll}B_{3} & B_{4}\end{array}\right]\right.$, $\left.\left[\begin{array}{ll}C_{3} & C_{4}\end{array}\right]\right\}$ are linearly independent.

(2) Each $D$-vector is perpendicular to the corresponding $B$-vector.

(3) The vectors $\left[\begin{array}{ll}E_{1} & E_{2}\end{array}\right]$ and $\left[E_{3} E_{4}\right]$ are each linearly dependent on the corresponding $C$-vectors.

Symmetries. A computation similar to that in the proof of Prop. 5.1 yields:

Proposition 6.1. When $H^{2} \neq 1$, the only symmetries of the CMC system for timelike surfaces in $\mathbf{H}^{2,1}$ arise from rigid motions of $\mathbf{H}^{2,1}$ and Lorentzian rotations of the first-order adapted frames. When $H^{2}=1$, the space of symmetries depends (in the sense of Cartan-Kähler) on two arbitrary functions of one variable. 
Conservation laws. First we compute the space of conservation laws for the ideal $\mathcal{I}_{1}$. (This will, of course, be isomorphic to the space of conservation laws for $\mathcal{I}_{2}$.) Suppose that

$$
\Phi=Q\left[\eta_{2}^{1} \wedge \eta^{2}-\eta_{3}^{2} \wedge \eta^{1}+2 \eta^{1} \wedge \eta^{2}\right]+\eta^{3} \wedge \gamma
$$

is a closed form in $\mathcal{I}_{1}$. Setting

$$
d Q=Q_{1} \eta^{1}+Q_{2} \eta^{2}+Q_{3} \eta^{3}+Q_{4} \eta_{1}^{3}+Q_{5} \eta_{2}^{3}
$$

and computing $d \Phi \equiv 0 \bmod \eta^{3}$ shows that

$$
\gamma=\left(Q_{2}-2 Q_{5}\right) \eta^{1}+\left(Q_{1}+2 Q_{4}\right) \eta^{2}+Q_{5} \eta_{1}^{3}+Q_{4} \eta_{2}^{3} .
$$

Then the condition $d \Phi=0$ gives the first-order PDE

$$
Q_{3}=0
$$

and four additional second-order PDEs for $Q$. The compatibility conditions for this system lead to three additional equations; the resulting system is not involutive, so it must be prolonged. The prolonged system is involutive with last nonvanishing Cartan character $s_{1}=2$, so the space of solutions (and hence the space of conservation laws for each of $\mathcal{I}_{1}$ and $\mathcal{I}_{2}$ ) depends on two arbitrary functions of one variable. This is the expected result: the CMC system is variational, so by Noether's theorem there is a one-to-one correspondence between conservation laws and symmetries.

Next we compute the space of conservation laws for $\mathcal{J}$, using the coframing described above, i.e.,

$$
\begin{aligned}
\theta_{1} & =2 \eta^{3}, & \theta_{2} & =2 \bar{\eta}^{3}, \\
\omega^{1} & =\eta^{1}-\eta^{2}+\eta^{3}, & \omega^{3} & =\eta^{1}+\eta^{2}-\eta^{3}, \\
\omega^{2} & =\eta_{3}^{2}+\eta^{2}+\eta_{3}^{1}+\eta^{1}, & \omega^{4} & =\eta_{3}^{2}-\eta_{3}^{1}-\eta^{1}+\eta^{2}+\frac{2}{\lambda-1} \eta^{3} .
\end{aligned}
$$

Suppose that

$\Phi=\theta_{1} \wedge\left(P_{1} \omega^{1}+P_{2} \omega^{2}+P_{3} \omega^{3}+P_{4} \omega^{4}\right)+\theta_{2} \wedge\left(Q_{1} \omega^{1}+Q_{2} \omega^{2}+Q_{3} \omega^{3}+Q_{4} \omega^{4}\right)+R \theta_{1} \wedge \theta_{2}$

is a closed form in $\mathrm{J}$. Computing $d \Phi \equiv 0 \bmod \theta_{1}, \theta_{2}$ shows that $Q_{1}=-P_{1}$, $Q_{2}=-P_{2}, Q_{3}=P_{3}$, and $Q_{4}=P_{4}$. Next, computing $d \Phi \equiv 0 \bmod \omega^{3}, \omega^{4}, \theta_{1}-\theta_{2}$ shows that

$$
R=\frac{1}{2}\left(P_{1}+P_{3}\right)+\frac{1}{\lambda-1} P_{4} .
$$

Then the condition $d \Phi=0$ gives a system of 14 first-order PDEs for the four functions $P_{1}, P_{2}, P_{3}, P_{4}$. The compatibility conditions for this system lead to four additional equations; the resulting system is not involutive, so it must be prolonged. The prolonged system is involutive with last nonvanishing Cartan character $s_{2}=1$, so the space of solutions (and hence the space of conservation laws for $\mathcal{J}$ ) depends on one arbitrary function of two variables.

Because the Cartan-Kähler analysis does not give explicit expressions for the conservation laws for $\mathcal{I}_{1}$ and $\mathcal{I}_{2}$, it is difficult to determine whether the pullbacks of these spaces to $\mathcal{B}$ coincide. In any case, since the space of conservation laws for $\mathcal{J}$ is significantly larger than those for $\mathcal{I}_{1}$ and $\mathcal{I}_{2}$, the pullbacks cannot possibly span the entire space of conservation laws for $\mathcal{J}$. Again, the significance of the "extra" conservation laws for $\mathcal{J}$ is unclear, and we hope to explore this issue in the future. 


\section{FurTher RESUlts}

Characteristic systems and their derived flags. We now give some results relating the structure of the characteristics of the Monge-Ampère systems linked by a parametric Bäcklund transformation to the $G$-structure invariants derived in \$2.

Recall that $\mathcal{C}_{11}, \mathcal{C}_{12}$ denote the characteristic systems of $\mathcal{I}_{1}$ and $\mathcal{C}_{21}, \mathcal{C}_{22}$ denote those of $\mathcal{I}_{2}$, and we have numbered them so that $\mathcal{C}_{11} \equiv \mathcal{C}_{21}$ and $\mathcal{C}_{12} \equiv \mathcal{C}_{22}$ modulo $\mathcal{K}$.

Theorem 2. If the vectors $\left[B_{1} B_{2}\right],\left[\begin{array}{ll}E_{1} & E_{2}\end{array}\right]$ are both linearly dependent on $\left[\begin{array}{ll}C_{1} & C_{2}\end{array}\right]$ at each point of $\mathcal{G}$, but at least two of these three vectors are nonzero, then each of $\mathcal{C}_{11}$ and $\mathcal{C}_{21}$ contains a one-dimensional integrable subsystem. The analogous conditions on $\left[\begin{array}{ll}B_{3} & B_{4}\end{array}\right],\left[\begin{array}{ll}C_{3} & C_{4}\end{array}\right]$ and $\left[\begin{array}{ll}E_{3} & E_{4}\end{array}\right]$ imply that $\mathcal{C}_{12}$ and $\mathcal{C}_{22}$ each contain one-dimensional integrable subsystems.

Before proving this theorem, we will need to explore the implications of the structure equations (15) for the derivatives of the torsion coefficients $A_{i}, C_{i}$, and $D_{i}$.

Recall that $\mathcal{R}_{1}, \mathcal{R}_{2}$ denote the Cartan systems of $\mathcal{I}_{1}, \mathcal{I}_{2}$ on $\mathcal{G}$, and the pullbacks of the characteristic systems to $\mathcal{G}$ include $\pi_{1}^{*} \mathcal{C}_{11}=\left\{\theta_{1}, \omega^{1}, \omega^{2}\right\}, \pi_{2}^{*} \mathcal{C}_{21}=\left\{\theta_{2}, \widetilde{\omega}^{1}, \widetilde{\omega}^{2}\right\}$. The fact that the span $\left\{\theta_{2}, \widetilde{\omega}^{1}, \widetilde{\omega}^{2}\right\}$ is the pullback of a well-defined system on $\mathcal{M}_{2}$ implies that

$$
\left.\begin{array}{l}
d \widetilde{\omega}^{1} \equiv 0 \\
d \widetilde{\omega}^{2} \equiv 0
\end{array}\right\} \quad \bmod \theta_{2}, \widetilde{\omega}^{1}, \widetilde{\omega}^{2}, \Lambda^{2}\left(\mathcal{R}_{2}\right) .
$$

Substituting in $\widetilde{\omega}^{1}=\omega^{1}+D_{2} \zeta$ and $\widetilde{\omega}^{2}=\omega^{2}-D_{1} \zeta$ gives

$$
\left.\begin{array}{c}
d D_{1} \equiv D_{2} \alpha_{3}+D_{1}\left(\gamma-\alpha_{4}\right)-C_{2}\left(D_{3} \omega^{3}+D_{4} \omega^{4}\right) \\
-E_{2} \theta_{1}+D_{10} \theta_{2}+D_{11} \omega^{1}+D_{12} \omega^{2} \\
d D_{2} \equiv D_{1} \alpha_{2}+D_{2}\left(\gamma-\alpha_{1}\right)+C_{1}\left(D_{3} \omega^{3}+D_{4} \omega^{4}\right) \\
+E_{1} \theta_{1}+D_{20} \theta_{2}+D_{21} \omega^{1}+D_{22} \omega^{2}
\end{array}\right\} \bmod \zeta
$$

for some functions $D_{1 j}$ and $D_{2 j}$. (In fact, computing $d\left(d \theta_{2}\right)=0$ and reducing modulo $\theta_{2}$ and $\omega^{3}$ shows that $D_{12}-D_{21}=F_{2}$.) We can derive analogous formulas for $d D_{3}$ and $d D_{4}$ by working with $\mathcal{C}_{12}$.

Computing $d\left(d \omega^{1}\right)=0$ and $d\left(d \omega^{2}\right)=0$, and reducing modulo $\omega^{1}, \omega^{2}$ gives

$$
\begin{aligned}
d C_{1}= & C_{1}\left(\beta_{1}+\beta_{4}-\alpha_{1}\right)-C_{2} \alpha_{2}+\left(E_{1}-C_{1} F_{1}\right) \zeta+A_{2} B_{1} \theta_{1}-B_{1} \theta_{2} \\
& +C_{11} \omega^{1}+C_{12} \omega^{2}+C_{13} \widetilde{\omega}^{3}+C_{14} \widetilde{\omega}^{4} \\
d C_{2}=C_{2} & \left(\beta_{1}+\beta_{4}-\alpha_{4}\right)-C_{1} \alpha_{3}+\left(E_{2}-C_{2} F_{1}\right) \zeta+A_{2} B_{2} \theta_{1}-B_{2} \theta_{2} \\
& +C_{21} \omega^{2}+C_{22} \omega^{2}+C_{23} \widetilde{\omega}^{3}+C_{14} \widetilde{\omega}^{4}
\end{aligned}
$$

for some functions $C_{1 j}$ and $C_{2 j}$. Then computing $d\left(d \theta_{2}\right) \equiv 0 \bmod \theta_{2}$ gives

$$
d A_{2}=A_{2}\left(\beta_{1}+\beta_{4}-\alpha_{1}-\alpha_{4}\right)+A_{2} F_{2} \zeta+C_{2} \widetilde{\omega}^{1}-C_{1} \widetilde{\omega}^{2}+A_{20} \theta_{2}+A_{23} \omega^{3}+A_{24} \omega^{4}
$$

for some functions $A_{2 j}$.

Recall that the derived system of a Pfaffian system $\mathcal{C}$ is spanned by those 1forms in $\mathcal{C}$ whose exterior derivatives are congruent to zero modulo $\mathcal{C}$, i.e., are linear combinations of wedge products, each of which has a form in $\mathcal{C}$ as one of 
its factors. The derived system, denoted by $\mathcal{C}^{(1)}$, is equal to $\mathcal{C}$ if and only if $\mathcal{C}$ is Frobenius. The derived system of $\mathcal{C}^{(1)}$ is denoted by $\mathcal{C}^{(2)}$, and so on.

It is easy to see from the structure equations (151) that

$$
\pi_{1}^{*} \mathcal{C}_{11}^{(1)}=\left\{\omega^{1}-C_{1} \theta_{1}, \omega^{2}-C_{2} \theta_{2}\right\} .
$$

Similarly, using the derivative formulas (38), one computes that

$$
\pi_{2}^{*} \mathcal{C}_{21}^{(1)}=\left\{\widetilde{\omega}^{1}-\left(C_{1} / A_{2}\right) \theta_{2}, \widetilde{\omega}^{2}-\left(C_{2} / A_{2}\right) \theta_{2}\right\}
$$

It follows that

$$
d\left(\widetilde{\omega}^{i}-\left(C_{i} / A_{2}\right) \theta_{2}\right) \equiv 0 \bmod \widetilde{\omega}^{1}-\left(C_{1} / A_{2}\right) \theta_{2}, \widetilde{\omega}^{2}-\left(C_{2} / A_{2}\right) \theta_{2}, \Lambda^{2}\left(\mathcal{R}_{2}\right),
$$

and this implies the identities

$$
\begin{aligned}
& A_{2} D_{10}+C_{1} D_{11}+C_{2} D_{12}=D_{1} C_{22}-D_{2} C_{21}-D_{3} C_{24}+D_{4} C_{23}+E_{2}-C_{2} F_{1}, \\
& A_{2} D_{20}+C_{1} D_{21}+C_{2} D_{22}=D_{2} C_{11}-D_{1} C_{12}+D_{3} C_{14}-D_{4} C_{13}-E_{1}+C_{1} F_{1} .
\end{aligned}
$$

We are now ready to prove Theorem 2

Proof. By hypothesis, there exists a sub-bundle $\mathcal{G}^{\prime} \subset \mathcal{G}$ on which

$$
B_{1}=C_{1}=E_{1}=0 \text {, }
$$

and we will restrict all forms and functions to this sub-bundle. Then on $\mathcal{G}^{\prime}$,

$$
\pi_{1}^{*} \mathcal{C}_{11}^{(1)}=\left\{\omega^{1}, \omega^{2}-C_{2} \theta_{2}\right\}, \quad \pi_{2}^{*} \mathcal{C}_{21}^{(1)}=\left\{\widetilde{\omega}^{1}, \widetilde{\omega}^{2}-\left(C_{2} / A_{2}\right) \theta_{2}\right\} .
$$

Computing $d\left(d \omega^{1}\right)=0$ on $\mathcal{G}^{\prime}$ and reducing modulo $\omega^{1}, \omega^{2}$ gives

$$
\alpha_{2} \wedge\left(\theta_{1} \wedge\left(B_{2} \theta_{2}-E_{2} \zeta\right)+C_{2} \widetilde{\omega}^{3} \wedge \widetilde{\omega}^{4}\right) \equiv 0 \bmod \omega^{1}, \omega^{2} .
$$

Since $C_{2} \neq 0$ and at least one of $B_{2}, E_{2}$ is nonzero, the 2-form in parentheses has rank four, and it follows that $\alpha_{2} \equiv 0$ modulo $\omega^{1}, \omega^{2}$. In fact, substituting (41) into (39) shows that

$$
\alpha_{2}=\frac{C_{11} \omega^{1}+C_{12} \omega^{2}}{C_{2}}
$$

and that $C_{13}=C_{14}=0$. It now follows from (15) that $d \omega^{1} \equiv 0 \bmod \omega^{1}$. Thus, $\pi_{1}^{*} \mathcal{C}_{11}=\left\{\theta_{1}, \omega^{1}, \omega^{2}\right\}$ contains the integrable 1 -form $\omega^{1}$.

Furthermore, we compute that

$$
d \widetilde{\omega}^{1} \equiv 0 \bmod \widetilde{\omega}^{1}, \widetilde{\omega}^{2}-\left(C_{2} / A_{2}\right) \theta_{2},
$$

which implies that $\widetilde{\omega}^{1} \in \pi_{2}^{*} \mathcal{C}_{21}^{(2)}$. (Here, it is necessary to compute keeping the identities (40) in mind.) The dimension of this second derived system is upper semi-continuous, and bounded above by $\operatorname{dim} \mathcal{C}_{21}^{(1)}=2$. Hence we may assume that either $\mathcal{C}_{21}^{(2)}=\mathcal{C}_{21}^{(1)}$ on an open set in $\mathcal{M}_{2}$, in which case $\mathcal{C}_{21}^{(1)}$ is Frobenius and must contain an integrable 1 -form, or $\operatorname{dim} \mathcal{C}_{21}^{(2)}=1$ on an open set. In the latter case, then $\pi_{2}^{*} \mathcal{C}_{21}^{(2)}=\left\{\widetilde{\omega}^{1}\right\}$, and so necessarily

$$
d \widetilde{\omega}^{1} \equiv 0 \quad \bmod \widetilde{\omega}^{1}, \Lambda^{2}\left(\mathcal{R}_{2}\right)
$$

However, we compute that

$$
d \widetilde{\omega}^{1} \equiv D_{20}\left(\theta_{2}-\left(A_{2} / C_{2}\right) \widetilde{\omega}^{2}\right) \wedge \zeta \bmod \widetilde{\omega}^{1},
$$

so it follows in this case that $D_{20}=0$ and $\widetilde{\omega}^{1}$ is integrable. 
Corollary 7.1. If $\left[\begin{array}{ll}D_{1} & D_{2}\end{array}\right]$ is perpendicular to each of $\left[B_{1} B_{2}\right],\left[\begin{array}{ll}C_{1} & C_{2}\end{array}\right]$ and $\left[E_{1} E_{2}\right]$, and if at least two of those three vectors are nonzero, then the intersection of $\mathcal{C}_{11}$ and $\mathcal{C}_{21}$ contains a one-dimensional integrable subsystem. Analogous conditions on $\left[\begin{array}{ll}B_{3} & B_{4}\end{array}\right],\left[\begin{array}{ll}C_{3} & C_{4}\end{array}\right],\left[\begin{array}{ll}D_{3} & D_{4}\end{array}\right]$ and $\left[\begin{array}{ll}E_{3} & E_{4}\end{array}\right]$ imply that $\mathcal{C}_{12}$ and $\mathcal{C}_{22}$ share an integrable 1 -form.

Proof. As in the proof of the previous theorem, we may restrict to the sub-bundle $\mathcal{G}^{\prime}$ where $B_{1}=C_{1}=E_{1}$. Then $D_{2}=0$, and $\omega^{1}=\widetilde{\omega}^{1}$ is an integrable 1-form common to both systems.

Symmetric Bäcklund transformations. We will say that a parametric Bäcklund transformation $\left(\mathcal{P}, \mathcal{M}_{1}, \mathcal{M}_{2}\right)$ is symmetric if there is a vector field $w$ on $\mathcal{P}$ such that

(a) $w$ is a symmetry of each of the one-dimensional Pfaffian systems $\left\{\theta_{1}\right\},\left\{\theta_{2}\right\}$ and $\{\zeta\}$;

(b) $w$ is transverse to the leaves $\mathcal{B}_{\lambda}$;

(c) w has nonzero projections to $\mathcal{M}_{1}$ and $\mathcal{M}_{2}$.

Although the Bäcklund transformations for the sine-Gordon equation and for Goursat's equation are symmetric, the Bäcklund transformation for pseudospherical surfaces and the transformation for CMC surfaces with $H^{2} \neq 1$ are not symmetric, since the only relevant symmetries of the underlying systems arise from rigid motions of the ambient space, and these do not change the Bäcklund parameter. The same argument cannot be applied to the CMC system with $H= \pm 1$, since in that case the space of symmetries (excluding rotations of first-order adapted frames) is not finite-dimensional, but depends on two functions of one variable.

Proposition 7.2. The parametric Bäcklund transformation of $₫[6$ for surfaces of constant mean curvature $H= \pm 1$ is not symmetric.

Sketch of the proof. Let $\mathrm{w}$ be a vector field on $\mathcal{P}$ and let $\left.\mathrm{w}\lrcorner \omega^{i}=x^{i}, \mathrm{w}\right\lrcorner \omega_{j}^{i}=y_{j}^{i}$ and $w\lrcorner d r=f$. Condition (a) above is equivalent to

$$
\begin{aligned}
\mathcal{L}_{\mathrm{w}} d u & =g_{0} d u, \\
\mathcal{L}_{\mathrm{w}} \eta^{3} & =g_{1} \eta^{3}, \\
\mathcal{L}_{\mathrm{w}} \bar{\eta}^{3} & =g_{2} \bar{\eta}^{3}
\end{aligned}
$$

for some functions $g_{0}, g_{1}, g_{2}$. Regard these equations as defining a rank three Pfaffian system on $\mathcal{P} \times \mathbb{R}^{10}$, with $f$ and the $g_{i}, x^{i}, y_{j}^{i}$ as the extra variables. The integrability conditions for this Pfaffian system imply that $f=0$, and hence there are no vector fields that satisfy both conditions (a) and (b).

\section{CONCLUDing REMARKS}

There are many issues raised here that merit further exploration. These include:

- In all of the examples computed here, the $E$-vectors and $C$-vectors are linearly dependent, and the $B$-vectors and $D$-vectors are perpendicular. Is this true in general? If not, what is the significance of these conditions?

- What is the significance of the "extra" conservation laws for the system $\mathcal{J}$ on $\mathcal{B}$ appearing in the sine-Gordon and CMC examples?

- Is there a condition on the invariants of the $G$-structure that indicates whether or not a Bäcklund transformation is symmetric? 
- What is the relationship between the invariants defined here and the contact invariants of $\mathcal{I}_{1}$ and $\mathcal{I}_{2}$ as hyperbolic Monge-Ampère systems? In particular, what necessary conditions does the existence of a parametric Bäcklund transformation impose?

We hope to explore these issues in future papers.

\section{REFERENCES}

[1] R. Bryant, S.-S. Chern, R. Gardner, P. Griffiths, H. Goldschmidt, Exterior Differential Systems, MSRI Publications, Springer, 1989.

[2] R. Bryant, P. Griffiths, Characteristic Cohomology of Differential Systems (II): Conservation Laws for a Class of Parabolic Equations, Duke Math. J. 78 (1995), 531-676. MR96d:58158

[3] R. Bryant, P. Griffiths and L. Hsu, Hyperbolic exterior differential systems and their conservation laws, Part 1, Selecta Mathematica, New Series 1 (1995) 21-112. MR97d:58008

[4] S.-S. Chern, C.-L. Terng, An analogue of Bäcklund's theorem in affine geometry, Rocky Mountain Math J. 10 (1980), 105-124. MR81h:58004

[5] J.N. Clelland, A Bäcklund transformation for timelike surfaces of constant mean curvature in $\mathbb{R}^{1,2}$, Bäcklund and Darboux Transformations. The Geometry of Solitons, 141-150, CRM Proc. Lecture Notes 29, Amer. Math. Soc., Providence, RI, 2001. MR2002j:53011

[6] J. N. Clelland, Homogeneous Bäcklund transformations of hyperbolic Monge-Ampère systems, Asian J. Math. 6 (2002), 433-480. MR2003j:58002

[7] P. Drazin, N. Johnson, Solitons: an introduction, Cambridge, 1989.

[8] E. Goursat, Leçons sur l'intégration des équations aux dérivées partielles du second ordre, vol. II, Gauthier-Villars, 1890.

[9] E. Goursat, Recherches sur quelques équations aux dérivées partielles du second ordre, $A n$ nales de la Faculté de Toulouse, deuxième serie 1 (1899), 31-78.

[10] S. Igonin, J. Krasil'shchik, On one-parametric families of Bäcklund transformations, Adv. Stud. Pure Math., vol. 37, Math. Soc. Japan, Tokyo, 2002.

[11] T.A. Ivey, J.M. Landsberg, Cartan for Beginners: Differential Geometry via Moving Frames and Exterior Differential Systems, Graduate Studies in Mathematics, American Mathematical Society, 2003.

[12] B. O'Neill, Elementary Differential Geometry, 2nd edition, Academic Press, 1997.

[13] _ Semi-Riemannian Geometry, Academic Press, 1983. MR85f:53002

[14] G. Penn-Karras, Klassification linearer Weingartenfläche in Raumformen, dissertation, Technische Universität Berlin, 1999.

[15] C. Rogers, Bäcklund transformations in soliton theory, in "Soliton theory: a survey of results", ed. A. P. Fordy, St. Martin's Press, 1990.

[16] C. Rogers, W. Shadwick, Bäcklund Transformations and Their Applications, Academic Press, 1982. MR 84c:58002

[17] M.Y. Zvyagin, Second order equations reducible to $z_{x y}=0$ by a Bäcklund transformation, Soviet Math. Dokl. 43 (1991) 30-34. MR.93a:35010

[18] M. Wadati, H. Sanuki, and K. Konno, Relationships among inverse method, Bäcklund transformation and an infinite number of conservation laws. Progr. Theoret. Phys. 53 (1975), 419-436. MR51:7516

Department of Mathematics, 395 UCB, University of Colorado, Boulder, Colorado 80309-0395

E-mail address: Jeanne.Clelland@colorado.edu

Department of Mathematics, College of Charleston, 66 George St., Charleston, South Carolina 29424-0001

E-mail address: IveyT@cofc.edu 